\title{
VARIETIES OF TOPOLOGICAL ALGEBRAS
}

\author{
WALTER TAYLOR
}

(Received 5 December 1975, revised 13 May 1976)

By a variety of topological algebras we mean a class $\mathscr{V}$ of topological algebras of a fixed type closed under the formation of subalgebras, products and quotients (i.e. images under continuous homomorphisms yielding the quotient topology). In symbols, $\mathscr{V}=\boldsymbol{S} \mathscr{V}=\boldsymbol{P} \mathscr{V}=\boldsymbol{Q} \mathscr{V}$. If $\mathscr{V}$ is also closed under the formation of arbitrary continuous homomorphic images, then $\mathscr{V}$ is a wide variety. As an example we have the full variety $\mathscr{V}=\operatorname{Mod}_{\tau}(\Sigma)$, the class of all topological algebras of a fixed type $\tau$ obeying a fixed set $\Sigma$ of algebraic identities. But not every wide variety is full, e.g. the class of all indiscrete topological algebras of a fixed type; in fact, as Morris observed (1970b), there exists a proper class of varieties of topological groups.

In our main results we present two ways of analyzing varieties, one "internal" and one "external". Our internal results assume that $\mathscr{V}$ has permutable congruences (see \$2) and follow closely the corresponding results for groups given by Brooks, Morris and Saxon (1973). For any $W \subseteq \mathscr{V}$, we prove that the Hausdorff topological algebras in QSP $W$ (the smallest variety $\supseteq W$ ) are precisely those in

\section{SPQSF W}

(where $\boldsymbol{F} W$ denotes the class of products of finitely many algebras from $W$ ) (see Theorem 4.1). A similar representation is given for Hausdorff algebras in $\boldsymbol{H S P} W$. Counterexamples will show that no results of this style can hold for varieties of topological lattices or semigroups.

Our external results are new even for varieties of topological groups. We prove that every wide variety $\mathcal{W}$ is definable by a set $\Sigma$ of (ordinary algebraic) laws and a class of limit laws, i.e. infinitary expressions of the form

$$
\tau_{d} \rightarrow \tau
$$

where $\left\{\tau_{d}: d \in D\right\}$ is a net of terms and $\tau$ is a term ("polynomial symbol") in the language of $W$. (See Theorem 6.2.) I.e., $W$ is precisely the class of 
topological algebras $\mathscr{A}$ which obey $\Sigma$ and which obey each limit law (*) in the sense that the net $\tau_{d}[\theta]$ approaches $\tau[\theta]$ in $\mathscr{A}$ for any assignment $\theta$ of $\mathscr{A}$-values to the variables appearing in $\tau_{d}$ and $\tau$. For some examples of limit laws, the reader should consult $\S 8$. Similar but more complicated results hold for varieties. These results descend from G. Birkhoff's theorem (1935) that every $\boldsymbol{H S P}$-closed class of (discrete) algebras is definable by a set of equations.

As remarked above, many topological wide varieties have a proper class of wide subvarieties, and hence there is no possible cardinal bound on the class of limit laws required, and in fact it seems likely that some wide varieties may require a proper class of limit laws, although we have no example. See Theorems 9.1 and 9.2 below.

In $\$ 0$ we give some basic lemmas about topological algebras and varieties, and in $\$ 1$ we review some facts about free algebras which are essentially well known. In $\$ 2$ and $\$ 3$ we discuss the companion algebraic properties of congruence-permutability and congruence-regularity and show some of their consequences for topological algebra. The main internal (representation) results are in $\$ 4$, with some counterexamples in $\$ 5$. The definability of wide varieties by limit laws is in $\$ 6$, and the corresponding result for varieties in $\S 7$. Examples of limit laws are in $\$ 8$, and in $\S 9$ we discuss when varieties form a proper class.

The results here were announced in Taylor $(1975 \mathrm{a}, \mathrm{b})$.

The author wishes to thank his sponsors the University of Colorado and the Fulbright Foundation, and his host institution, the University of New South Wales, for making possible this research. And special thanks to S. A. Morris who introduced the author to this material and provided a large amount of assistance and encouragement. Various helpful remarks came from P. G. Dixon, K. Keimel and R. N. McKenzie.

\section{Preliminaries}

$\left(A, \mathcal{U}, F_{t}\right)_{t \in T}$ is a topological algebra if $(A, \mathscr{U})$ is a topological space and for each $t \in T$ there exists an integer $n_{t}$ such that $F_{t}$ is a continuous function $F_{t}: A^{n_{t}} \rightarrow A$ (relative to the topology $\mathcal{U}$ on $A$ and its $n_{t}$-fold product on $A^{n_{t}}$ ). The type of $\left(A, \mathcal{U}, F_{t}\right)_{t \in T}$ is $\left(n_{t}\right)_{t \in T}$; whenever we speak of a class of topological algebras, we mean that they all have the same type.

A (continuous) homomorphism $f: A \rightarrow B$ between two topological algebras $\mathscr{A}=\left(A, \mathscr{U}, F_{t}\right)_{t \in T}$ and $\mathscr{B}=\left(B, \mathscr{W}, G_{t}\right)_{t \in T}$ of the same type is a continuous function $f:(A, \mathscr{U}) \rightarrow(B, \mathcal{W})$ such that $f F_{t}\left(a_{1}, \cdots, a_{n_{t}}\right)=G_{t}\left(f a_{1}, \cdots, f a_{n_{t}}\right)$ for every $t \in T$ and all $a_{1}, \cdots, a_{n_{t}} \in A$. If $f$ is onto, then we say $\mathscr{B}$ is a 
lomomorphic image of $\mathscr{A}$, and if $f$ is also (topologically) a quotient map, then ve say that $\mathscr{B}$ is a quotient of $\mathscr{A}$. If $f$ is a bijection with continuous inverse, hen $\mathscr{A}$ and $\mathscr{B}$ are isomorphic $(\mathscr{A} \cong \mathscr{B}) . \mathscr{A}$ is a (closed) subalgebra of $\mathscr{B}$ iff $\downarrow \subseteq \mathscr{B}$ and $(A, \mathscr{U})$ is a (closed) subspace of $(B, \mathscr{U})$. Products of topological lgebras are formed by placing the usual product topological and algebraic tructures on the product of the underlying sets. If $\Omega$ is any class of topological lgebras we define

$\boldsymbol{H} \Omega=$ the class of all homomorphic images of members of $\Omega$;

$Q \Omega=$ the class of all quotients of members of $\Omega$;

$\overline{\boldsymbol{Q}} \Omega=$ the class of all Hausdorff quotients of members of $\Omega$;

$S \Omega=$ the class of all algebras isomorphic to subalgebras of members of $\Omega$;

$\overline{\boldsymbol{S}} \Omega=$ the class of all algebras isomorphic to closed subalgebras of members of $\Omega$;

$\boldsymbol{P} \Omega=$ the class of all products of families of members of $\Omega$;

$F \Omega=$ the class of all products of finite families of members of $\Omega$.

The next two propositions have completely standard proofs, which we mit. Here $\Omega$ denotes an arbitrary class of topological algebras. The ypothesis of the first proposition is always fulfilled for congruenceermutable varieties $\mathscr{V}$ (see $\S 2$ and $\S 4$ ). This is the only case in which we will se this proposition.

Proposition 0.1. If $\mathscr{V}$ is a variety in which all quotient maps are open taps, and $\Omega \subseteq \mathcal{V}$, then the smallest variety $\supseteq \Omega$ is $\mathbf{Q S P} \Omega$.

Proposition 0.2. The smallest wide variety $\supseteq \Omega$ is $\boldsymbol{H S P} \Omega$.

Sometimes $\boldsymbol{Q S P} \Omega[\boldsymbol{H S P} \Omega]$ is called the [wide] variety generated by $\Omega$.

The following proposition in the case of groups, and a similar proof, were iscovered several years ago by I. D. Macdonald. See also Lemima 2.7 of Iorris (1969).

Proposition 0.3. If $\mathscr{A}$ and $\mathscr{B}$ are algebraically isomorphic and $\mathscr{B}$ has the idiscrete topology, then $\mathscr{B} \in \boldsymbol{Q S P} \mathscr{A}$.

Proof. We assume that $\mathscr{A}$ and $\mathscr{B}$ have the same underlying algebra $\left.\mathcal{A}, F_{t}\right)_{t \in T}$. Let $\mathscr{C}$ be the topological subalgebra of $\mathscr{A}^{\boldsymbol{N}_{1}}$ consisting of all :quences $\left(a_{1}, a_{2}, \cdots\right)$ which are eventually constant, and map $\mathscr{C} \rightarrow \mathscr{B}$ by apping each sequence to its ultimate constant value. Clearly this map is a ontinuous homomorphism; to finish we need only see that it is an open map nd hence a quotient map - see e.g. Kelley (1955, p. 95)). But a nonempty ssic open subset of $\mathscr{A}^{\boldsymbol{N}_{11}}$ is of the form 


$$
U=U_{1} \times \cdots \times U_{k} \times \mathscr{A} \times \mathscr{A} \times \cdots
$$

which contains sequences of arbitrary eventual constant value. Hence $U$ maps onto $\mathscr{B}$.

COROLlaRy 0.4. Let $\mathscr{V}$ be any variety and $\mathscr{V}_{0}$ the class of indiscrete algebras in $\mathscr{V}$. Then $\mathscr{V}_{0}$ is a subvariety of $\mathscr{V}$ which is algebraically the same as $\mathscr{V}$, i.e. every algebra in $\mathscr{V}$ is algebraically isomorphic to some algebra in $\mathscr{V}_{0}$.

Note that Corollary 0.4 also follows immediately from the main Theorem 7.1, for clearly an indiscrete algebra obeys every contingent limit law. Since $\boldsymbol{Q}$, $\boldsymbol{S}$ and $\boldsymbol{P}$ coincide on indiscrete algebras with their algebraic meanings, we immediately obtain the next corollary, which is needed to obtain $\Sigma$ in the main Theorem.

Corollary 0.5. If $\mathscr{V}$ is any variety of topological algebras, then

$$
\overrightarrow{\mathcal{V}}=\left\{\left(A, F_{t}\right)_{i \in T}: \exists \mathscr{U}\left(A, \mathcal{U}, F_{t}\right)_{t \in T} \in \mathscr{V}\right\}
$$

is a variety of algebras.

Not much general topology is required for reading this paper beyond the basic ideas of convergence of nets. The general algebra required is also rather basic; the special topics of congruence-permutability and congruence. regularity are discussed below. Following Morris (1969) we will sometimes use barred letters $\overline{\mathscr{A}}, \overline{\mathscr{V}}, \bar{X}$, to indicate non-topological notions, e.g. algebras instead of topological algebras, sets instead of spaces. Occasionally we denot $\epsilon$ ordinary algebras $\mathfrak{A}, \mathfrak{B}, \mathfrak{C}$ as well.

\section{Free topological algebras}

The following proposition is of a type which has become quite standarc and well known, especially under the name of "adjoint functor theorems' (see any book on category theory). Its proof is little different from Birkhoff': (1935) construction of free algebras. We assume that the reader is familia with the algebraic ideas of free algebras, terms, and so forth (see Cohn (1965 or Grätzer (1968)).

Proposition 1.1. If $\mathscr{V}$ is any variety (in fact if $\boldsymbol{S} \mathscr{V}=\boldsymbol{P} \mathscr{V}=\mathscr{V}$ ) and $X \boldsymbol{i}$ any space, then there exists $\mathscr{A} \in \mathscr{V}$ and continuous $f: X \rightarrow \mathscr{A}$ such that whenever $g: X \rightarrow \mathscr{B}$ is continuous with $\mathscr{B} \in \mathscr{V}$, there exists a (*) unique continuous homomorphism $\bar{g}: \mathscr{A} \rightarrow \mathscr{B}$ such that $\bar{g} \circ f=g$

and moreover if $f^{\prime}: X \rightarrow \mathscr{A}^{\prime}$ also obeys (*) (with $f, \mathscr{A}$ replaced by $\left.f^{\prime}, \mathscr{A}^{\prime}\right)$, the there exists an isomorphism $F: \mathscr{A} \rightarrow \mathscr{A}^{\prime}$ with $F \circ f=f^{\prime}$. 
As usual, $\mathscr{A}$ is called $\mathscr{V}$-free on $X$, denoted $F_{\mathcal{V}}(X)$. In the next result, we will see that in fact $f$ is one-one, and algebraically $\mathscr{A}$ looks just like an ordinary free algebra. We let $\bar{X}$ be the underlying set of the space $X$ and $\bar{V}$ the variety of algebras given by the indiscrete algebras in $\mathscr{V}$ (see 0.5 ).

Proposition 1.2. The free algebra $F_{\bar{v}}(\bar{X})$ has a topology $U$ so that in 1.1 we may take $\mathscr{A}=\left(F_{\bar{v}}(\bar{X}), \mathcal{U}\right)$ and $f$ the identity function, i.e. $\left(F_{\bar{v}}(\bar{X}), \mathcal{U}\right)$ is the free algebra $F_{v}(X)$.

Corollary 1.3. $\overline{F_{V}(\bar{X})}=F_{\bar{r}}(\bar{X})$.

Proof of 1.2. Take $f: X \rightarrow \mathscr{A}$ as in 1.1 , and extend algebraically to $f: F_{\bar{x}}(\bar{X}) \rightarrow \overline{\mathscr{A}}$ (using 0.3 to see that $\overline{\mathscr{A}} \in \overline{\mathscr{V}}$ ). Regarding $F_{\bar{V}}(\bar{X})$ as an indiscrete algebra in $\mathscr{V}$, we have continuous $g: X \rightarrow F_{\bar{V}}(\bar{X})$ with $g=$ identity, and so, applying 1.1 , we have $\bar{g}: \mathscr{A} \rightarrow F_{\bar{v}}(\bar{X})$ with $\bar{g} \circ f=$ identity. We need to see that also $f \circ \bar{g}=$ identity. To see this, note that

$$
f \circ \bar{g} \circ f=f \circ 1=f=1 \circ f,
$$

and thus $f \circ \bar{g}=1$ (by the uniqueness assertion in (*), taking 1 for $g$ ).

And so from now on, we always mean by $F_{V}(X)$ a topological algebra with $X \rightarrow F_{2}(X)$ the identity function. (Warning: the identity function here is continuous but not necessarily an embedding of spaces, e.g. in case $\mathscr{V}$ is indiscrete.) But there are some cases in which $X \rightarrow F_{\mathscr{V}}(X)$ is an embedding. Here 2 denotes the two-element discrete space.

PROPOSITION 1.4. If the variety $\mathscr{V}$ does not consist entirely of indiscrete algebras and the space $X$ is embeddable in some Cantor space $2^{\prime}$, then $X \rightarrow F_{V}(X)$ is an embedding.

Proof. By standard arguments, it is enough to show that some $\mathscr{A} \in \mathcal{V}$ has a subspace homeomorphic to $X$. And by $\boldsymbol{S}$ and $\boldsymbol{P}$, it is enough to show that some $\mathscr{A} \in \mathscr{V}$ has a two-element discrete subspace. We know there exists $\mathscr{B} \in \mathscr{V}$ with a proper open subset $U$, i.e. for some $a, b \in B, a \in U$ and $b \notin U$. But then one easily checks that $\{(a, b),(b, a)\}$ is a two-element discrete subspace of $\mathscr{B}^{2}$.

The next proposition is largely due to Świerczkowski (1964); for a proof of this version, see Taylor (to appear). For varieties of groups, this theorem is due to Markov (1945).

Proposition 1.5. If $X$ is a metrizable space with metric $d_{0}$ and $\overline{\mathcal{V}}$ is any variety of algebras, then there exists a metric $d$ on the (algebraic) free algebra $\bar{F}_{\bar{v}}(\bar{X})$ such that 
(1) $d$ is an extension of $d_{0}$;

(2) all operations of $\bar{F}_{\bar{Y}}(\bar{X})$ are continuous in the d-topology;

(3) for any $\alpha \in \bar{F}_{\bar{V}}(\bar{X})$ and sufficiently small $\varepsilon$, the $\varepsilon$-neighborhood of $\alpha$ consists of all $\beta \in \bar{F}_{\bar{r}}(\bar{X})$ for which there exist $x_{1}, \cdots, x_{n}, y_{1}, \cdots, y_{n} \in \bar{X}$ and a term $F$ such that

$$
\begin{aligned}
& \alpha=F\left(x_{1}, \cdots, x_{n}\right), \\
& \beta=F\left(y_{1}, \cdots, y_{n}\right) \text { and } \\
& \sum_{i=1}^{n} d_{0}\left(x_{i}, y_{i}\right)<\varepsilon .
\end{aligned}
$$

COROLlaRY 1.6. If $\mathscr{V}$ is a full variety of topological algebras and $X$ is a metrizable space, then $X$ is embedded as a subspace of $F_{V}(X)$.

But, $n . b$. , the topology of $F_{v}(X)$ may have more open sets than those given by the Świerczkowski topology on $\bar{F}_{\bar{v}}(\bar{X})$. For the free topological group on $X$ cannot be metrizable unless $X$ is discrete [S. A. Morris, private communication]. For more information on free topological algebras, consult e.g. Burgin (1972).

\section{Permutability of congruence}

An algebra $\mathfrak{A}$ has permutable congruences iff $\theta \circ \varphi=\varphi \circ \theta$ for any congruences $\theta, \varphi$ on $\mathfrak{A}$. (Here $\theta \circ \varphi$ is the relational product $\{(a, c):(\exists b \in$ If $)((a, b) \in \theta$ and $(b, c) \in \varphi)\}$.) A consequence of congruence-permutability is that $\theta \circ \varphi$ is itself transitive, and hence a congruence, the join $\theta \vee \varphi$. More generally, if always

$$
\theta \circ \varphi \circ \theta \circ \varphi \circ \cdots=\varphi \circ \theta \circ \varphi \circ \theta \circ \cdots
$$

with $k$ factors on each side, then $\mathfrak{A}$ has $k$-permutable congruences. And we say a variety $\mathscr{V}$ has $k$-permutable congruences if each of its algebras does. A theorem of Malcev (1954) states that a variety $\mathscr{V}$ has permutable congruences iff there exists a term $p(\cdot, \cdot, \cdot)$ in the language of $\mathscr{V}$ such that the equations

$$
x=p(y, y, x)=p(x, y, y)
$$

hold identically in $\mathscr{V}$. (And so groups have permutable congruences, using $p(x, y, z)=x y^{-1} z$.) Permutability of congruences had a tremendous influence in the early development of general algebra. particularly in Jordan-Hölder type theorems and theorems on direct product representations - see Cohn (1965), or Chaps. 5-8 of Crawley and Dilworth (1973) or the algebra chapter in Birkhoff (1948), where many historical remarks are given. An algebra with permutable congruences has a modular congruence lattice (Birkhoff (1948)), 
as does one with 3-permutable congruences (Jónsson (1953)) (but not necessarily for $k$-permutable for $k \geqq 4$ ). And $k$-permutability of congruences has appeared in topological algebra in the theorem that every component of a topological algebra in a congruence- $k$-permutable variety has commutative homotopy (Taylor (to appear)).

Permutability of congruences allows us to form quotients, given arbitrary congruences $\theta$ on topological algebras. If $\theta$ is an equivalence relation on $A$, and $B \subseteq A$, by the $\theta$-saturation of $B$ we mean

$$
\{a \in A:(\exists b \in B) a \theta b\},
$$

i.e. the union of the $\theta$-blocks meeting $B$.

THEOREM 2.1. A full variety $\mathcal{V}$ has permutable congruences if and only if for any open set $U$ of any $\mathscr{A} \in \mathscr{V}$ and any congruence $\theta$ on $\mathscr{A}$, the $\theta$-saturation of $U$ is also open.

Proof. Malcev proved the direction $(\rightarrow)$ (1954). The converse direction $(\leftarrow)$ was discovered by J. Hagemann (preprint) [Our proof in fact shows that if $\gamma$ is not congruence-permutable, then the condition fails for some Hausdorff $\mathscr{A} \in \in^{\prime} \mathscr{Y}$. Hagemann's $\mathscr{A}$ is not Hausdorff]; here we give another proof. Take $[0,1]$ to be the unit interval with the usual topology, and let the free algebra $F_{l}([0,1])$ be topologized with the Świerczkowski metric described in 1.5 above. Choose $\varepsilon<\frac{1}{2}$ and small enough $>0$ so that the $\varepsilon$-neighborhood of 0 in $F_{\gamma}([0.1])$ is as described in condition 3 of Theorem 1.5 above, and let $U$ be the $\varepsilon$-neighborhood of 0 . Take $\theta$ to be the congruence generated by $(0,1)$ - note that

$$
\alpha \theta \beta \Leftrightarrow \varphi(\alpha)=\varphi(\beta),
$$

where $\varphi: F_{y}([0,1]) \rightarrow F_{y}([0,1))$ is given by mapping 1 to 0 and all other generators identically. The $\theta$-saturation of $U$ is an open set containing 1 , and so there exists $t \in[0,1]$ and $\alpha \in F_{1}([0,1])$ such that

(a) $1-\varepsilon<t<1$;

(b) $\alpha \theta t$;

(c) $\alpha \in U$.

Now by $1.5(3)$ and (c), we have

(d) $0=q\left(x_{1}, \cdots, x_{n}\right)$

(e) $\alpha=q\left(y_{1}, \cdots, y_{n}\right)$

(f) $\quad \sum_{i=1}^{n}\left|x_{i}-y_{i}\right|<\varepsilon$

for some $x_{1}, \cdots, x_{n}, y_{1}, \cdots, y_{n} \in[0,1]$. Thus by (a) and (f), there exists $c$, $0<c<t$, such that for $1 \leqq i \leqq n$. either $x_{i}<c$ and $y_{i}<c$ or $x_{i}>c$ and $y_{i}>c$. Now form $p(x, y, z)$ from $q\left(y_{1}, \cdots, y_{n}\right)$ by simultaneously substituting $x$ for 
each occurrence of $y_{i}<c, y$ for 1 and $z$ for the remaining $y_{j}$ in $q\left(y_{1}, \cdots, y_{n}\right)$. By choice of $c, p(x, z, z)$ can be formed from $q\left(x_{1}, \cdots, x_{n}\right)$ by substituting $x$ for each $x_{i}<c$ and $z$ for each $x_{i}>c$. And so from (d) we obtain

$$
p(x, z, z)=x
$$

holding identically in $\mathscr{V}$. Now by (e), (b) and (*),

$$
q\left(\varphi y_{1}, \cdots, \varphi y_{n}\right)=\varphi q\left(y_{1}, \cdots, y_{n}\right)=\varphi \alpha=\varphi t=t .
$$

In this equation we substitute $x$ for variables $<c$ and $z$ for those $>c$. From the definitions of $c, p$ and $\varphi$, it is clear that we obtain

$$
p(x, x, z)=z \text {. }
$$

We have now obtained the two Malcev equations for congruencepermutability.

COROLlaRY 2.2. If $\mathscr{A}$ is in some congruence-permutable full variety and $\theta$ is any congruence on $\mathscr{A}$, then there exists a unique topology on $\mathscr{A} / \theta$ so that $\mathscr{A} / \theta$ is a topological algebra and $\mathscr{A} \rightarrow \mathscr{A} / \theta$ is an open continuous map.

Proof. Uniqueness is easy. To see existence, define a subset of $\mathscr{A} / \theta$ to be open iff its inverse image is open in $\mathscr{A}$. The map $\mathscr{A} \rightarrow \mathscr{A} / \theta$ is then open by Theorem 2.1 . We illustrate the continuity of the operations with one binary operation + . If we want to check the continuity of + at $\left([a]_{\theta},[b]_{\theta}\right)$, we consider an arbitrary neighborhood of $[a+b]_{\theta}$, which we may take to be of the form $U / \theta$, for $U$ open in $\mathscr{A}$. By continuity of + on $\mathscr{A}$, there exist open sets $V, W, a \in V, b \in W$ with $V+W \subseteq U$. Since $\theta$ is a congruence, $V / \theta+W / \theta \subseteq U / \theta$, and $V / \theta$ and $W / \theta$ are open, since $\mathscr{A} \rightarrow \mathscr{A} / \theta$ is an open map.

Notation. $/ \theta$ will mean the topological algebra uniquely specified by Corollary 2.2 .

It is to be emphasized that in Corollary 2.2 one needs the congruencepermutability of QSPAA, not just $\mathscr{A}$ alone.

The next lemma is due to Findlay (1960).

LEMMA 2.3. If $V$ is a congruence-permutable variety and $\theta \subseteq \mathscr{A}^{2} \in \mathscr{V}$ is a subalgebra of $\mathscr{A}^{2}$ containing the diagonal subalgebra $\{(a, a): a \in \mathscr{A}\}$, then $\theta$ is transitive.

Proof. If $(a, b),(b, c) \in \theta$, then

$$
(a, c)=p((a, b),(b, b),(b, c)) \in \theta,
$$

where $\boldsymbol{p}$ is the Malcev polynomial described above. 
CoRollary 2.4. If $\mathscr{V}$ is a congruence-permutable variety, $\mathscr{A} \subseteq \mathscr{B} \in \mathscr{V}$ and $\theta$ is a congruence on $\mathscr{A}$, then its closure $\bar{\theta}$ is a congruence on $\overline{\mathscr{A}} \subseteq \mathscr{B}$.

Proof. Clearly $\bar{\theta}$ is a subalgebra of $\mathscr{B}^{2}$, and $\bar{\theta}$ contains the diagonal of $\bar{A}$, and hence is transitive by 2.3 . The closure of a symmetric relation is always symmetric.

We now let $\bar{\Delta}$ be the closure of the identity relation (in any given algebra), and let $\mathscr{A} / \bar{\Delta}$ be the quotient algebra given by 2.2 and 2.4 . The next corollary is easy and we omit its proof.

COROLLARY 2.5. If $\mathscr{A}$ is in some congruence-permutable variety, then $\mathscr{A} / \bar{\Delta}$ is a Hausdorff quotient of $\mathscr{A}$ which is maximal in the sense that if $\mathscr{B}$ is Hausdorff and $f: \mathscr{A} \rightarrow \mathscr{B}$ a continuous homomorphism, then f factors through $\mathscr{A} \rightarrow \mathscr{A} / \bar{\Delta}$.

Proposition 2.6. If $\mathscr{A}$ is in some congruence-permutable variety, $a, b \in \mathscr{A}$ and there exists an open set $W$ with $a \in W, b \notin W$, then there exist disjoint open neighborhoods of $a$ and $b$.

Proof. Since $p$ (see the beginning of this section) is continuous and

$$
p(a, b, b)=a,
$$

there exist open sets $U, V$ with $a \in U, b \in V$, such that

$$
p[U, V, b] \subseteq W .
$$

We claim $U \cap V=\varnothing$; otherwise, for some $c \in U \cap V$, we would have

$$
b=p(c, c, b) \in W,
$$

a contradiction.

COROLlaRY 2.7. Every $T_{0}$ topological algebra in a congruence-permutable variety is Hausdorff.

Corollary 2.8. ( $\mathscr{A}, \bar{\Delta}$ as in 2.5.) For all $a, b \in \mathscr{A}, a \bar{\Delta} b$ if and only if $a$ and $b$ are in exactly the same open sets of $\mathscr{A}$.

Corollary 2.9. ( $\mathscr{A}, \bar{\Delta}$ as in 2.5.) Every open set of $\mathscr{A}$ is $\bar{\Delta}$-saturated.

Corollary 2.10. For $\mathscr{A}$ as above, the natural map $\mathscr{A} \rightarrow \mathscr{A}_{0} \times \mathscr{A} / \bar{\Delta}$ is a topological and algebraic embedding (where $\mathscr{A}_{0}$ is $\mathscr{A}$ with the indiscrete topology).

COROLlARY 2.11. If $\mathscr{V}$ is a congruence-permutable variety, then.

$$
\mathscr{V}=\boldsymbol{S P}\left(\mathscr{V} \cap\left(T_{2} \cup \text { Indiscrete }\right)\right) \text {. }
$$

(For varieties of groups, this was noticed by Morris (1970b, Theorem 6.7).) 
The study of indiscrete algebras may be regarded as pure algebra, and will not be discussed in this paper. The Hausdorff part of certain varieties will be discussed in $\$ 4$.

Proposition 2.12. If $\mathscr{A}$ is in some congruence-permutable variety, $\mathscr{B}$ is a dense subalgebra of $\mathscr{A}, \theta$ is a closed congruence on $\mathscr{B}$ and $\bar{\theta}$ is the closure of $\theta$ in $\mathscr{A}^{2}$, then

$$
\mathscr{B} / \theta \in S\{\mathscr{A} / \bar{\theta}\}
$$

ProOf. Let $\varphi: \mathscr{A} \rightarrow \mathscr{A} / \bar{\theta}$ be the natural map, which is open and continuous. Obviously it is enough to establish that $\varphi \mid B$ is continuous, open on to its image and has kernel $\theta$. The continuity is immediate, and since $\theta$ is closed, $\bar{\theta} \cap \mathscr{B}^{2}=\theta$. Thus we need only check that $\varphi \mid \mathscr{B}$ is an open map onto $\varphi[\mathscr{B}]$. To establish this we will prove that

$$
\varphi[\mathscr{B} \cap U]=\varphi[\mathscr{B}] \cap \varphi[U]
$$

for every open set $U$ of $\mathscr{A}$, which is enough, since $\varphi$ is open. The inclusion $\subseteq$ of $(*)$ is immediate. To see the reverse inclusion $\supseteq$, we take $x \in \varphi[\mathscr{B}] \cap \varphi[U]$ and prove that $x \in \varphi[\mathscr{B} \cap U]$. We have

$$
x=[b]_{\bar{\theta}}=[u]_{\bar{\theta}}
$$

for some $b \in \mathscr{B}, u \in U$. Since $p$ (see the beginning of this section) is continuous and

$$
p(b, b, u)=u
$$

there exist open sets $W, V$ with $b \in W, u \in V$ such that

$$
p[b, W, V] \subseteq U .
$$

Since $(b, u) \in \bar{\theta}$, there exists $(\beta, \gamma) \in \theta$ with $\beta \in W, \gamma \in V$. And so clearly

$$
\alpha=p(b, \beta, \gamma) \in \mathscr{B} \cap U
$$

moreover $\alpha \theta p(b, \beta, \beta)=b$, and thus $\varphi(\alpha)=x$, that is, $x \in \varphi[\mathscr{B} \cap U]$.

We next show that sometimes in congruence-permutable varieties one can replace $\boldsymbol{Q}$ by $\overline{\boldsymbol{Q}}$ and $\boldsymbol{S}$ by $\overline{\boldsymbol{S}}$ (i.e. one can restrict one's attention to Hausdorff quotients and closed subalgebras). These last two corollaries will be applied at the finish of the proof of 4.1 below. They are essential for 4.5 .

COROLI.ARY 2.13. If $\Omega$ is any subclass of a congruence-permutable variety, then

$$
\bar{Q} \boldsymbol{S} \Omega \subseteq \boldsymbol{S} \overline{\mathbf{Q}} \overline{\boldsymbol{S}} \Omega
$$


Proof. If $\mathscr{C} \in \overline{\boldsymbol{Q}} \boldsymbol{S} \Omega$, then we may take $\mathscr{C}=\mathscr{B} / \boldsymbol{\theta}, \mathscr{B} \subseteq \mathscr{D} \in \Omega$, with $\theta$ a closed congruence on $\mathscr{B}$. Taking $\mathscr{A}=\mathscr{\mathscr { B }}$, the closure of $\mathscr{B}$ in $\mathscr{D}$, the result is immediate from the previous proposition.

COROLlary 2.14. For $\Omega$ as above

$$
T_{2} \cap \boldsymbol{S P} \Omega \subseteq \boldsymbol{S P} \overline{\boldsymbol{Q}} \Omega .
$$

Proof. If $\mathscr{A}$ is a Hausdorff algebra in $\boldsymbol{S P} \Omega$, then we have $\mathscr{A} \subseteq \Pi \mathscr{B}_{i}$ (each $\mathscr{B}_{i} \in \Omega$ ). Take $\bar{\Delta}$ as defined just before 2.5 , and consider the natural map

$$
f: \mathscr{A} \rightarrow \prod\left(\mathscr{B}_{i} / \bar{\Delta}\right) \text {. }
$$

We first prove that $f$ is one-one. If $\alpha \neq \beta, \alpha, \beta \in \mathscr{A}$. then $\alpha$ and $\beta$ have disjoint basic neighborhoods, which easily implies that for some $i$ there exist disjoint open sets $U_{i}, V_{i}$ with $\alpha_{i} \in U_{i}, \beta_{i} \in V_{i}$. Thus $\left(\alpha_{i}, \beta_{i}\right) \notin \bar{\Delta}$ and so $f(\alpha) \neq f(\beta)$. Finally we prove that $f$ is an embedding. If $\alpha_{d}$ is a net not approaching $\alpha$, we need to see that $f\left(\alpha_{d}\right)$ is a net not approaching $f(\alpha)$, i.e. that for some $i,\left[a_{d i}\right]_{j}$ does not approach $\left[a_{i}\right]_{\Sigma}$. Clearly $\alpha$ has a basic neighborhood $U=U_{i_{1}} \times \cdots \times U_{i_{k}} \times \Pi \mathscr{B}_{j}\left(j \neq i_{1}, \cdots, i_{k}\right)$ such that $\alpha_{d}$ is not eventually in $U$. But then clearly $\alpha_{d}$ is not eventually in some subbasic neighborhood $U_{i} \times \Pi \mathscr{B}_{j}(j \neq i)$ of $\alpha$. Thus by Corollary $2.9,\left[a_{d i}\right]_{5}$ is not eventually in the neighborhood $\left[U_{i}\right]_{j}$ of $\left[\alpha_{i}\right]_{\Sigma}$. Thus $\left[\alpha_{d i}\right]_{\Sigma}$ does not approach $\left[\alpha_{i}\right]_{\bar{s}}$.

\section{Regularity of congruences}

We say that an algebra :' has regular congruences if any two congruences on $?$ t having one block in common are equal. A variety $\mathscr{V}$ has regular congruences if every, $\mathcal{I} \in \mathcal{q}$ has regular congruences. For the history of this notion see e.g. Grätzer (1970)). Csákány (1970) proved that $\mathscr{V}$ is congruenceregular if and only if there are ternary terms $F_{1}, \cdots, F_{k}$ in the language of $y$ such that $\mathscr{V}$ satisfies

$$
\forall x y z\left[x=y \leftrightarrow \bigwedge_{i=1}^{k}\left(F_{i}(x, y, z)=z\right)\right] .
$$

(In the variety of groups, which is easily seen to be congruence-regular, we may take $k=1$ and $F_{1}(x, y, z)=x y{ }^{1} z$.) Similar results were given by Grätzer (1970) and Wille (1970). Congruence-regularity and congruence-permutability (\$2) are independent properties, but Hagemann has shown (to appear) that every congruence-regular variety has $k$-permutable congruences for some $k$ and also has modular congruence lattices.

Our main use of congruence-regularity is to limit the size of discrete subalgebras of products. (See Corollary 3.4 and its application in Theorem 
9.1 below.) Congruence-regularity appeared before in topological algebra in our theorem (Taylor, 1974) that every infinite compact Hausdorff algebra in a congruence-regular variety has power $\geqq 2 \aleph^{\aleph_{0}}$. We repeat a lemma used for that result, giving it a new proof, since the proof there used a general result of Hagemann, which is now known to be false.

LemMA 3.1. If $\mathscr{A}$ has an isolated point and $\mathscr{A}$ is in some congruenceregular variety, then $\mathscr{A}$ is discrete.

Proof. Let $F_{1}, \cdots, F_{k}$ be as in (*), and let $a$ be an isolated point. To see that any $b \in \mathscr{A}$ is isolated, since each $F_{i}$ is continuous,

$$
F_{i}(b, b, a)=a \quad(1 \leqq i \leqq k)
$$

and $\{a\}$ is open, there exist open sets $U_{i}(1 \leqq i \leqq k)$ such that $b \in U_{i}$ and

$$
F_{i}\left[U_{i}, U_{i}, a\right]=\{a\} \quad(1 \leqq i \leqq k) .
$$

We claim that $\{b\}=U_{1} \cap \cdots \cap U_{k}$ and hence is open. For if $c \in U_{1} \cap \cdots$ $\cap U_{k}$, then

$$
F_{i}(b, c, a)=a \quad(1 \leqq i \leqq k),
$$

and so, by $(*), b=c$.

Let $\mathscr{V}_{F}$ denote the full variety which is algebraically the same as $\mathscr{V}$. Note that, as with congruence-permutability, $\mathscr{V}$ is congruence-regular if and only if $\mathscr{V}_{F}$ is. If $\Pi_{i \in I} \mathscr{A}_{i}$ is any product and $J \subseteq I$, we let $\pi_{J}: \Pi_{i \in I} \mathscr{A}_{i} \rightarrow \Pi_{i \in J} \mathscr{A}_{i}$ denote the natural projection. Our results will deal with the following properties of a variety $\mathscr{V}$ :

$\boldsymbol{R}(\mathscr{V}): \mathscr{V}$ has regular congruences.

$I(\mathscr{V}):$ if $\mathscr{A} \in \mathscr{V}$ and $\mathscr{A} \subseteq \mathscr{B}^{\prime}$ contains an isolated point, then there exists finite $J \subseteq I$ such that $\pi_{J}$ is one-one on $\mathscr{A}$.

$\boldsymbol{K}(\mathscr{V})$ : if $\mathscr{A} \in \mathscr{V}$ and $\mathscr{A} \subseteq \Pi_{i \in I} \mathscr{B}_{i}$ contains an isolated point, then there exists finite $J \subseteq I$ such that $\pi_{J}$ is one-one on $\mathscr{A}$.

$I_{\mathrm{m}}(\mathscr{V})$ : if $\mathscr{A} \in \mathscr{V}, \mathscr{A} \subseteq \mathscr{B}^{I}, \mathscr{A}$ a $T_{1}$-algebra in which one point has a neighborhood basis of $\leqq m$ open sets, then there exists $J \subseteq I,|J|=m$, and $\pi_{J}$ is one-one on $\mathscr{A}$. ( $\mathrm{m}$ any cardinal.)

$\boldsymbol{K}_{\mathrm{m}}(\mathscr{V}):$ if $\mathscr{A} \in \mathscr{V}, \mathscr{A} \subseteq \Pi_{i \in I} \mathscr{B}_{i}, \mathscr{A}$ a $T_{1}$-algebra in which one point has a neighborhood basis of $\leqq m$ open sets, then there exists $J \subseteq I,|J| \leqq m$, and $\pi_{J}$ is one-one on ( $m$ any cardinal.)

$D(\mathscr{V}):$ if $\mathscr{A} \in \mathscr{V}, \mathscr{A} \subseteq \Pi_{i \in I} \mathscr{B}_{i}, \mathscr{A}$ discrete, then there exists finite $J \subseteq I$ such that $\pi_{J}$ is one-one on $\mathscr{A}$.

Note that $\boldsymbol{D}(\mathscr{V})$ may be modified just as $I(\mathscr{V})$ was, but we omit these conditions since little is known about them. 
THeOREM 3.2. If $\mathscr{V}$ is a full variety, then $\boldsymbol{R}(\mathscr{V}), \mathbf{I}(\mathscr{V}), \boldsymbol{I}_{\mathrm{m}}(\mathscr{V}), \boldsymbol{K}(\mathscr{V})$ and $\boldsymbol{K}_{\mathrm{m}}(\mathscr{V})$ are all equivalent (for any $\mathrm{m}$ ).

Proof. We first show $\boldsymbol{K}(\mathscr{V}) \rightarrow I(\mathscr{V}) \rightarrow \boldsymbol{R}(\mathscr{V}) \rightarrow \boldsymbol{K}(\mathscr{V})$. The first implication is a fortiori. For the next, let us assume that $\mathscr{V}$ does not have regular congruences, and disprove $I(\mathscr{V})$. Thus we are given congruences $\theta, \varphi$ on $\mathfrak{S} \in \mathscr{V}$ which are unequal but have a block in common. Thus $\mathfrak{B}=\mathfrak{E} / \theta \wedge \varphi$ is an algebra in $\mathscr{V}$ with a congruence $\psi=\theta \vee \varphi$ which has a singleton block $\{c\}$ and a non-singleton block $D=\{a, b, \cdots\}(a \neq b)$. Take $\mathscr{B}$ to be $\mathscr{B}$ equipped with the discrete topology, take an infinite index set $I$, and let $\mathscr{A}$ be the subalgebra of $\mathscr{B}^{I}$ generated by

$$
\{(c, c, c, \cdots)\} \cup D^{I} \subseteq \mathscr{B}^{I} .
$$

Obviously no finite set of coördinates can separate points of $\mathscr{A}$, and so to show that $I(\mathscr{V})$ fails, it is enough to show that $\langle c, c, \cdots\rangle$ is isolated in $\mathscr{A}$. It is enough to show that $\vec{c}=\langle c, c, \cdots\rangle$ is determined by its first coördinate, i.e. that if $x=\left\langle c, x_{2}, x_{3}, \cdots\right\rangle$, then $x=\vec{c}$. We may represent $x=p\left(\vec{c}, \alpha_{1}, \cdots, \alpha_{n}\right)$ for $p$ some term and $\alpha_{1}, \cdots, \alpha_{n} \in D^{I}$. In each coordinate $i \in I$, since $D$ is a $\psi$-block,

$$
x_{i}=p\left(c, \alpha_{1 i}, \cdots, \alpha_{n i}\right) \psi p\left(c, \alpha_{10}, \cdots, \alpha_{n 0}\right)=c .
$$

And so $x_{i}=c$ (since $\{c\}$ is a $\psi$-block), i.e. $x=\vec{c}$.

To see $\boldsymbol{R}(\mathscr{V}) \rightarrow \boldsymbol{K}(\mathscr{V})$, we assume that $\mathscr{A}$ is in the congruence-regular variety $\mathscr{V}$ and that $\alpha$ is an isolated point of $\mathscr{A} \subseteq \Pi_{i \in I} \mathscr{B}_{i}$. This means that for some finite $J \subseteq i,\{\alpha\}$ is a singleton block in the congruence on $\mathscr{A}$ given by $x \boldsymbol{\theta} y$ iff $\pi_{J} x=\pi_{J} y$. By congruence-regularity, every block of $\theta$ is a singleton, i.e. $\pi_{J}$ is one-one on $\mathscr{A}$.

The implications $\boldsymbol{K}_{\mathrm{m}}(\mathscr{V}) \rightarrow \boldsymbol{I}_{\mathrm{m}}(\mathscr{V}) \rightarrow \boldsymbol{R}(\mathscr{V}) \rightarrow \boldsymbol{K}_{\mathrm{m}}(\mathscr{V})$ have similar proofs which we omit.

Corollary 3.3. In any variety $\mathscr{V}, \boldsymbol{R}(\mathscr{V}) \rightarrow \boldsymbol{K}(\mathscr{V})$.

COROLlaRy 3.4. If $\mathscr{V}$ is congruence-regular and $\mathscr{A} \subseteq \Pi_{i \in I} \mathscr{B}_{i} \in \mathscr{V}$ and $\mathscr{A}$ has an isolated point, then $|\mathscr{A}| \leqq \boldsymbol{N}_{0}+\left|\mathscr{B}_{i}\right|$ for some $i$.

(Discrete closed subsets of powers have been studied for example in Mycielski (1964).)

Note that in fact, in Corollary $3.4, \mathscr{A}$ must actually be discrete, by Lemma 3.1. As far as $D(\mathscr{V})$ is concerned, we do not have so much to say. Clearly $\boldsymbol{I}(\mathscr{V}) \rightarrow \boldsymbol{D}(\mathscr{V})$ but not conversely. $\boldsymbol{D}(\mathscr{V})$ follows from weak regularity of congruences, by a proof very similar to that of the relevant part of 3.2. (For weak regularity, see $\$ 6$ of Taylor (1973) or Hagemann (to appear).) Finally, 
we state without proof the following theorem - a class of varieties is said to be Malcev-definable if it is defined by the existence of polynomials obeying certain laws (for example, like Malcev's characterization of permutability of congruences mentioned in \$2). And congruence-regularity is also Malcevdefinable - see Csákány (1970), Grätzer (1970), Wille (1970) — in fact, the Csákány conditions (*) are easily converted to equations in any fixed variety. For more details, see Taylor (1973); the proof of 3.5 resembles many proofs in $\$ 5$ of Taylor (1973). It is a non-constructive proof, not yielding explicit polynomials. For a general treatment of Malcev-definability, see Baldwin and Berman (to appear), Neumann (1974) or Taylor (1973).

\section{Theorem 3.5. D( $\left.\mathcal{Y}^{\prime}\right)$ is Malcev-definable.}

We close $\$ 3$ with an example showing that $\boldsymbol{D}(\mathscr{V})$ does not follow from the congruence-permutability of $\mathscr{V}$ (defined in $\$ 2$ ), and that Corollary 3.4 fails if congruence-regularity is replaced by congruence-permutability.

TheOREM 3.6. There exists a congruence-permutable variety $\mathcal{I}$ and discrete algebras $\mathscr{A}_{\alpha} \in \mathcal{V}$ ( $\alpha$ any ordinal) such that for any $\alpha$

$$
\begin{aligned}
& \mathscr{A}_{\alpha} \subseteq \prod_{\beta<\alpha} \mathscr{A}_{\beta+1}, \text { and } \\
& \left|\mathscr{A}_{\alpha}\right|=|\alpha| .
\end{aligned}
$$

In particular, for $\alpha=\boldsymbol{N}_{1}, \mathcal{A}_{x}$ is an uncountable discrete subalgebra of a product of countable algebras.

Proof. $\mathcal{F}$ has one ternary operation $F$ and is defined by the laws

$$
\begin{aligned}
& F(x, z, z)=x \\
& F(x, x, z)=z,
\end{aligned}
$$

which entail congruence-permutability, by Malcev's theorem of $\$ 2$. For any $\alpha$, the discrete algebra $\mathscr{A}_{\alpha}$ has universe $\alpha=\{\eta: \eta<\alpha\}$. with $F: A_{\alpha}{ }^{3} \rightarrow A_{\alpha}$ defined via the above laws together with the condition

$$
\begin{aligned}
& F(x, y, x)=y \\
& F(x, y, z)=\min \{x, y, z\}, \text { where } x, y, z \text { are distinct. }
\end{aligned}
$$

For $\alpha>\beta$ one easily checks that the mapping $\theta_{\beta}^{\alpha}: \mathscr{A}_{\alpha} \rightarrow \mathscr{A}_{\beta+1}$ given by

$$
\theta_{\beta}^{\alpha}(\gamma)=\beta \wedge \gamma
$$

is a homomorphism. Now one easily checks that if $\beta+1<\alpha$, then $\beta$ is the unique solution in $\mathscr{A}_{\alpha}$ of the equation 


$$
\theta_{\beta+1}^{\alpha}(x)=\beta,
$$

and if $\beta+1=\alpha$, then $\beta$ is the unique solution in $\mathscr{A}_{\alpha}$ of the equation

$$
\theta_{\beta}^{\alpha}(x)=\beta \text {. }
$$

Thus every member of $\mathscr{A}_{\alpha}$ is specified by a single one of the maps $\theta_{\beta}^{\alpha}$, and so the family of maps $\left\{\theta_{\beta}^{\alpha}: \beta<\alpha\right\}$ embeds $\mathscr{A}_{\alpha}$ as a discrete subalgebra of $\prod_{\beta<\alpha} \mathscr{A}_{\beta+1}$.

R. N. McKenzie [personal communication] has recently improved 3.6 by finding a discrete subalgebra with power $2^{N_{0}}$ of a product of countable algebras in a congruence-permutable variety. Moreover he proved that this example is best possible in the sense that $2^{*}$ cannot be made larger. His results will appear elsewhere. As we will see in Corollary 5.4 below, discrete sublatices of products of countable lattices can be arbitrarily large.

\section{Representation of Hausdorff algebras in varieties}

Here are our main "internal" results on varieties. For congruencepermutability, see $\$ 2$. Some such hypothesis is needed, as will be seen by examples in $\$ 5$.

THEOREM 4.1. If $\Omega$ is any subclass of a congruence-permutable variety of topological algebras, then

$$
T_{2} \cap \boldsymbol{Q S P} \Omega=\boldsymbol{S P} \overline{\mathbf{Q S}} \boldsymbol{F} \Omega .
$$

Corollary 4.2. If $\Omega$ is as above, then

$$
\boldsymbol{Q S P} \Omega=\boldsymbol{S P}(\mathscr{X} \cup \overline{\boldsymbol{Q S}} \bar{F} \Omega),
$$

where $\mathscr{X}$ is the indiscrete variety generated by the algebras in $\Omega$ taken with the indiscrete topologies.

THEOREM 4.3. If $\Omega$ is any subclass of a congruence-3-permutable variety of topological algebras, then every Hausdorff algebra in HSP $\Omega$ is algebraically in SPHSF $\Omega$.

Proofs. Clearly 4.2 is immediate from 4.1 and 2.11 . We give the proofs of 4.1 and 4.3 together, due to their similarity. In 4.1 we need only prove the inclusion $\subseteq$. And so we consider $\Omega \subseteq$ some 3-permutable $\mathscr{V}$ and Hausdorff $\mathscr{A} \in \boldsymbol{H S P} \Omega$, given precisely by

$$
\mathscr{B}, \in \Omega(i<\kappa)(\kappa \text { a fixed ordinal })
$$

$$
\mathscr{B} \subseteq \prod_{i<\kappa} \mathscr{B}_{i}
$$




$$
g: \mathscr{B} \rightarrow \mathscr{A} \text {, a continuous homomorphism. }
$$

For $\sigma$ a finite subset of $\kappa$, we let $\theta_{\alpha}$ be the congruence on $\mathscr{B}$ defined by

$$
(\alpha, \beta) \in \theta_{\sigma} \quad \text { iff } \quad(\forall i \in \sigma) \quad\left(\alpha_{i}=\beta_{i}\right)
$$

(i.e. $\theta_{\sigma}=\operatorname{ker} \pi_{\sigma}$ ). And let

$$
\theta=\operatorname{ker} g=\{(\alpha, \beta): g \alpha=g \beta\} \subseteq \mathscr{B}^{2} .
$$

Proceeding algebraically for the moment, let $\Sigma$ be the collection of all finite subsets of $\kappa$ and consider the natural map

$$
f: \mathscr{B} \rightarrow \prod_{\sigma \in \Sigma} \mathscr{B} / \theta \vee \theta_{\sigma}
$$

We claim that

$$
\operatorname{ker} f=\theta=\operatorname{ker} g \text {. }
$$

Clearly $\theta \subseteq \operatorname{ker} f$. To see the reverse inclusion, we assume by way of contradiction that $f(\alpha)=f(\beta)$ but $g(\alpha) \neq g(\beta)$. Since $\mathscr{A}$ is Hausdorff and $g$ is continuous, there exist open sets $U, V$ with $\alpha \in U, \beta \in V$ and

$$
(U \times V) \cap \theta=\varnothing .
$$

We may assume without loss of generality that

$$
\begin{aligned}
& U=\mathscr{B} \cap\left(U_{0} \times \cdots \times U_{m} \times \prod_{i>m} \mathscr{B}_{i}\right), \text { and } \\
& V=\mathscr{B} \cap\left(V_{0} \times \cdots \times V_{m} \times \prod_{i>m} \mathscr{B}_{i}\right)
\end{aligned}
$$

for some finite $m$ and open sets $U_{i}, V_{i}(0 \leqq i \leqq m)$. Taking $\tau=\{0, \cdots, m\}$, since $f(\alpha)=f(\beta)$, we have $(\alpha, \beta) \in \theta \vee \theta_{r}$, and hence by 3-permutability of congruences

$$
\alpha \theta_{\tau} \gamma \theta \delta \theta_{\tau} \beta
$$

for some $\gamma, \delta \in \mathscr{B}$. By inspection of the product form of $U, V$, and by definition of $\theta_{r}$, we see that $\gamma \in U, \delta \in V$, and hence $(\gamma, \delta) \in(U \times V) \cap \theta$, which contradicts the fact that $(U \times V) \cap \theta=\varnothing$. And thus (5) is proved.

And so there exists a unique one-one homomorphism $\varphi$ making this diagram commute

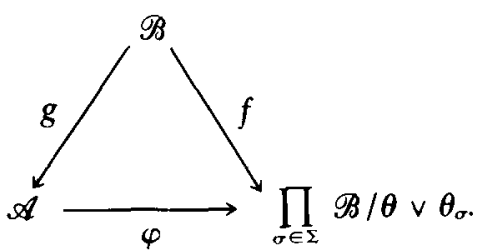


Clearly $\mathscr{B} / \boldsymbol{\theta}_{\sigma} \in \boldsymbol{S F} \Omega$ and so its homomorphic image $\mathscr{B} / \boldsymbol{\theta} \vee \boldsymbol{\theta}_{\sigma} \in \boldsymbol{H S F} \Omega$. Thus algebraically $\mathscr{A} \in \boldsymbol{S P H S F} \Omega$, and Theorem 4.3 is proved.

To prove 4.1, we continue this argument, with the strengthened assumptions that $\mathscr{V}$ is congruence-permutable and $g$ is a quotient map. And so by Theorem $2.1, g$ is in fact an open map. We next supply each $\mathscr{B} / \theta \vee \theta_{\sigma}$ with the quotient topology given by 2.2 , so that clearly each $\mathscr{B} / \boldsymbol{\theta} \vee \boldsymbol{\theta}_{\sigma} \in \boldsymbol{Q S F} \Omega$. It is evident, with these topologies, since $g$ is onto and $f$ is continuous, that $\varphi$ is continuous.

We next proceed to prove that $\varphi$ is an embedding. For this it is enough to check that $f$ is an open map of $\mathscr{B}$ onto its image. It is enough to check $f[\mathscr{B} \cap U]$, for $U$ a basic open subset of $\Pi \mathscr{B}_{i}$, which is w.l.o.g.

$$
U=U_{0} \times \cdots \times U_{m} \times \prod_{i>m} \mathscr{B}_{i}
$$

for some finite $m$ and open sets $U_{i}(0 \leqq i \leqq m)$. Taking $\tau=\{0, \cdots, m\}$, we claim that

$$
f[\mathscr{B} \cap U]=f[\mathscr{B}] \cap\left\{U /\left(\theta \vee \theta_{\tau}\right) \times \prod_{\sigma \neq \tau} \mathscr{B} / \theta \vee \theta_{\sigma}\right\},
$$

which clearly implies that $f[\mathscr{B} \cap U]$ is open. The inclusion $\subseteq$ is immediate, and so we check only $\supseteq$. Taking $x$ in the R.H.S., we first have $x=f(\alpha)$ for some $\alpha \in \mathscr{B}$. And so in the $\tau$ th component, we have $x_{\tau}=[\alpha]_{\theta_{v} \theta_{r}}$. But also $x_{\tau}=[u]_{\theta \vee \theta_{\tau}}$ for some $u \in U$, and thus

$$
(\alpha, u) \in \theta \vee \theta_{\tau} .
$$

By congruence-permutability, there exists $\beta \in \mathscr{B}$ such that

$$
(\alpha, \beta) \in \theta \quad \text { and } \quad(\beta, u) \in \theta_{r} .
$$

Since $\theta=\operatorname{ker} f$, we have $f(\beta)=f(\alpha)=x$, and the form of $U$ implies that $\beta \in U$. Thus $x \in f[\mathscr{B} \cap U]$, and the claim is verified. Thus $\varphi$ is an embedding.

And so we have proved that

$$
\mathscr{A} \in \mathbf{S P Q S F} \Omega .
$$

Applying Corollary 2.14, we see that

$$
\begin{aligned}
\mathscr{A} & \in \boldsymbol{S P} \bar{Q} Q S F \Omega \\
& =S P \bar{Q} S F \Omega,
\end{aligned}
$$

and applying Corollary 2.13 we see that

$$
\begin{aligned}
\mathscr{A} & \in \boldsymbol{S P S} \overline{Q S F} \Omega \\
& =S P \bar{Q} \bar{S} F \Omega .
\end{aligned}
$$


As remarked in the introduction, the above proof follows closely the lines of Brooks, Morris and Saxon (1973), who proved 4.1 for varieties of topological groups, and who discovered the following corollary in the case of groups. Note the stark contrast with the algebraic case, where $\mathscr{V}=$ $\boldsymbol{H S P}\left\{F_{1}\left(\boldsymbol{N}_{0}\right)\right\}$.

COROLlaRY 4.4. If $\mathscr{V}$ is a congruence-3-permutable full variety which is not (algebraically speaking) residually small, then there exists no set $\Omega$ of topological algebras with $\mathscr{V}=\boldsymbol{H S P} \Omega$.

Note. A variety $\mathscr{V}$ is residually small if there exists a set $K$ so that $\mathscr{V}=\boldsymbol{S P K}$. The variety of all groups is not residually small, for there exist arbitrarily large simple groups. For more information, consult Taylor (1972).

Proof. Immediate, because by 4.3 , such a $K$ would be given by HSF $\Omega$.

COROLLARY 4.5. If $\mathscr{V}$ is a congruence-permutable variety, then the class of subalgebras of compact algebras in $\mathscr{V}$ is also a variety. And in the case of countable type, this class is $\mathbf{S P C _ { 0 }}$; where $C_{0}$ is the set of compact metric algebras in $\mathscr{V}$ together with the class of subdirectly irreducible indiscrete algebras in $\mathscr{V}$.

Proof. Let $\Omega$ be the class of compact algebras in $\mathscr{V}$, and note that $\boldsymbol{P} \overline{\mathbf{Q}} \overline{\boldsymbol{S}} \boldsymbol{\Omega}=\boldsymbol{\Omega}$. Thus a Hausdorff algebra in $\boldsymbol{Q S P} \Omega$ is in $\boldsymbol{S P} \overline{\mathbf{Q}} \overline{\boldsymbol{F}} \boldsymbol{F} \Omega=\boldsymbol{S} \Omega$, and thus embeddable in a compact algebra. And of course, every indiscrete algebra is compact. Thus by Corollary 2.11, $\boldsymbol{Q S P} \Omega \subseteq \boldsymbol{S} \Omega$, and so $\boldsymbol{S} \Omega$ is a variety. The final sentence is immediate from Theorem 7.7 of Taylor (1971).

(The author's earlier proof of this corollary was improved by S. A. Morris.)

\section{Some counterexamples}

Here we show that the theorem of Brooks, Morris and Saxon, generalized to congruence-permutable varieties in $\$ 4$, fails for lattices and semigroups.

We let $L$ denote the lattice

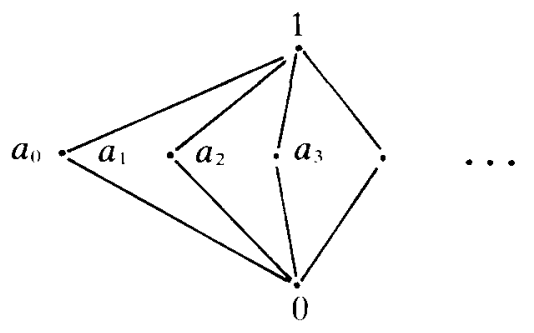


which has least element 0 , greatest element $1, \boldsymbol{N}_{0}$ atoms $a_{0}, a_{1}, a_{2}, \cdots$, and no other elements. And we let $S$ denote the idempotent semigroup with multiplication table

\begin{tabular}{l|lll}
\multicolumn{1}{c}{} & 0 & 1 & 2 \\
\cline { 2 - 4 } 0 & 0 & 0 & 0 \\
1 & 1 & 1 & 1 \\
2 & 0 & 1 & 2
\end{tabular}

Regard both $L$ and $S$ as having the discrete topology.

LEMMA 5.1. If $\sigma, \tau$ are lattice theoretic terms in $x_{0}, \cdots, x_{n}$ which are not identically equal in $L$, then there is an assignment $x_{i} \mapsto b_{i}$ making $\sigma \neq \tau$, with all $b_{i}$ chosen from the sublattice $L_{n}$ :

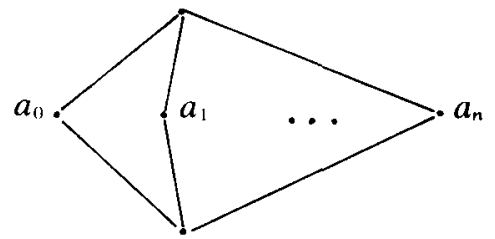

Proof. By symmetry.

The next corollary requires 5.1 in the case of $L$, but is automatic for the finite algebra $S$.

COROLlary 5.2. If $\mathscr{V}=\boldsymbol{H S P}\{L\}$ or $\boldsymbol{H S P}\{S\}$, then the finitely generated free algebras $F_{1}(n)$ are all finite.

LEMMA 5.3. For any set $K$ and lattice (resp. semigroup) term $\tau\left(x_{0}, \cdots, x_{n}\right)$ $\left(x_{0}, \cdots, x_{n} \in K\right)$, there exists a finite set $F$ of assignments $\theta: K \rightarrow L_{n}$ (resp. $S$ ) such that for any term $\sigma$, either $\sigma=\tau$ holds identically in $L$ (resp. $S$ ) or $\sigma[\theta] \neq \tau[\theta]$ for some $\theta \in F$.

Proof. For $L$, let $F$ consist of all $\theta$ such that each $\theta(\lambda) \in L_{n}$ and $\theta(\lambda)=\theta(\mu)$ whenever $\lambda, \mu \in K-\left\{x_{0}, \cdots, x_{n}\right\}$. For arbitrary $\sigma$, if $\sigma=$ $\sigma\left(x_{0}, \cdots, x_{n}\right)$, then there is obviously nothing to prove, and so we may assume that $\sigma$ depends on some $\lambda \notin\left\{x_{11}, \cdots, x_{n}\right\}$. Thus one has $\sigma(\vec{a}) \neq \sigma(\vec{b})$ with $\vec{a}$ differing from $\vec{b}$ only in the $\lambda$ th place. By monotonicity of lattice operations,

$$
\sigma\left(a_{0}, \cdots, a_{n}, 0,0, \cdots\right)<\sigma\left(a_{0}, \cdots, a_{n}, 1,1, \cdots\right) .
$$

And so $\sigma[\theta] \neq \tau[\theta]$ for either $\theta=\left(a_{0}, \cdots, a_{n}, 0,0, \cdots\right)$ or $\theta=$ $\left(a_{0}, \cdots, a_{n}, 1,1, \cdots\right)$. 
For $S$, let $F$ consist of all $\theta$ with $\theta(\lambda)=1$ for all $\lambda \notin\left\{x_{0}, \cdots, x_{n}\right\}$. An arbitrary $\sigma$ is just a product $\lambda_{1} \cdots \lambda_{k}$ (each $\lambda_{i} \in K$ ), and as before, we need only consider the case when some $\lambda_{i} \notin\left\{x_{0}, \cdots, x_{k}\right\}$. For this case we let $\theta\left(x_{i}\right)=2(0 \leqq i \leqq n)$ and $\theta(\lambda)=1 \quad\left(\lambda \notin\left\{x_{0}, \cdots, x_{n}\right\}\right)$. Clearly $\sigma[\theta]=1$ and $\tau[\theta]=2$.

We now take $\mathscr{A}=$ either $L$ or $S$ with the discrete topology and $I$ any set. Let $J=\mathscr{A}^{\mathscr{A} I}$ and for each $\alpha \in \mathscr{A}^{I}$ define $\bar{\alpha} \in \mathscr{A}^{\prime \cup J}$ via

$$
\begin{aligned}
& \bar{\alpha}(i)=\alpha(i) \quad(i \in I) \\
& \bar{\alpha}(j)=j(\alpha) \quad(j \in J) .
\end{aligned}
$$

We then define $\mathcal{M}$ to be the subalgebra of $\mathscr{A}^{I \cup J}$ generated by $\left\{\bar{\alpha}: \alpha \in \mathscr{A}^{I}\right\}$.

Corollary 5.4. $\mathcal{M}$ is discrete in $\mathscr{A}^{I \cup J}$, and $|\mathcal{M}| \geqq 2^{|I|}$.

Proof. Consider an arbitrary $b \in \mathcal{M}$. For some term $\tau$, we have

$$
b=\tau\left(\bar{\alpha}_{0}, \cdots, \bar{\alpha}_{n}\right) .
$$

Let $F$ be a set of assignments given by 5.3 , where we take $K=\mathscr{A}^{I}, x_{i}=\alpha_{i}$ $(i=0, \cdots, n)$. Of course $\theta: K \rightarrow \mathscr{A}$ is just a member of $J=\mathscr{A}^{K}$. We claim that $b$ is determined in $\mathcal{M}$ by $\left\langle b_{\theta}: \theta \in F\right\rangle$. For an arbitrary member of $\mathcal{M}$ is

$$
c=\sigma\left(\bar{\beta}_{0}, \cdots, \bar{\beta}_{m}\right) \text {. }
$$

Now if $c \neq b$, then $\sigma$ is not identically equal to $\tau$ on $\mathscr{A}$, and so, by 5.3 , $\sigma[\theta] \neq \tau[\theta]$ for some $\theta \in F$. But then

$$
\begin{aligned}
c_{\theta} & =\sigma\left(\bar{\beta}_{0}(\theta), \cdots, \bar{\beta}_{m}(\theta)\right) \\
& =\sigma\left(\theta\left(\beta_{0}\right), \cdots, \theta\left(\beta_{m}\right)\right) \\
& =\sigma[\theta],
\end{aligned}
$$

and similarly $b_{\theta}=\tau[\theta]$. Hence $b_{\theta} \neq c_{\theta}$.

Corollary 5.5. For $\mathscr{A}=L$ or $S$ and any set I let $\mathscr{A}^{\prime}$ denote the algebraic power of $\mathscr{A}$ with the discrete topology. Then $\mathscr{A}^{I} \in$ QSPA.

Proof. Clearly $\mathscr{A}^{1}$ is a homomorphic image of $\mathcal{M}$, algebraically. And $\mathcal{M}$ has the discrete topology.

For subdirectly irreducible algebras, and theorems on the limitation of the size of subdirect irreducibles in varieties, see e.g. Taylor (1972) and references given there.

THEOREM 5.6. If $\mathfrak{A}=L$ or $S$, then there exist arbitrarily large subdirectly irreducible algebras in the variety $\boldsymbol{H S P} \mathfrak{A}$. 
Proof. For $L$, this is well-known; in fact, all ultrapowers of $L$ are simple. For $S$ this is proved in $\S 3$ of Taylor (1976) (closely following work of Gerhard (1971)).

Corollary 5.7. If $\mathscr{A}=L$ or $S$ (with the discrete topology), then QSPAA contains arbitrarily large discrete subdirectly irreducible algebras.

Corollary 5.8. If $\mathscr{V}=Q S P L$ or $Q S P S$, and

$$
T_{2} \cap \mathscr{V} \subseteq \boldsymbol{S P X}
$$

then $X$ is a proper class.

Corollary 5.9. Theorem 4.1 fails for $\Omega=\{L\}$ or $\{S\}$.

\section{Limit laws}

For convenience we fix a type and fix a class $X$ of variables, disjoint from all other sets and classes under consideration. We let $(D, \leqq)$ be an arbitrary directed set and $f: D \rightarrow T e(X)$ a function from $D$ to the class of terms over $X$ of the given type. Usually we will denote the term $f(d)$ by $\tau_{d}$. And we let $\tau$ denote an arbitrary term. A limit law is an infinitary formal expression

$$
f \rightarrow \tau
$$

with $f$ and $\tau$ arbitrary as above; usually we do not mention $D$ and simply write

$$
\tau_{d} \rightarrow \tau,
$$

"the net of terms $\tau_{d}$ always approaches the term $\tau$ ". For various examples of limit laws, the reader is referrred to $\$ 8$ below. An assignment is a mapping $\theta: X \rightarrow \mathscr{A}$ where $\mathscr{A}$ is a topological algebra of the given type. We say that $\mathscr{A}$ satisfies the limit law $\tau_{d} \rightarrow \tau$ under the assignment $\theta$ if

$$
\tau_{d}[\theta] \rightarrow \tau[\theta]
$$

in the topology of $\mathscr{A}$. (I.e. the net $\tau_{d}[\theta]$ converges to $\tau[\theta]$, but not necessarily uniquely.) And we say that $\mathscr{A}$ satisfies $\tau_{d} \rightarrow \tau$ identically (synonymously, $\tau_{d} \rightarrow \tau$ holds identically in $\mathscr{A}$, or, $\mathscr{A}$ obeys the limit law $\tau_{d} \rightarrow \tau$ ), in symbols,

$$
\mathscr{A} \vDash \tau_{d} \rightarrow \tau,
$$

if $\mathscr{A}$ satisfies $\tau_{d} \rightarrow \tau$ under every assignment $\theta: X \rightarrow \mathscr{A}$. If $\Omega$ is any class of topological algebras, then

$$
\Omega \vDash \tau_{d} \rightarrow \tau
$$


will mean that $(*)$ holds for every $\mathscr{A} \in \Omega$. For $\Gamma$ any class of limit laws, the class of models of $\Gamma$ is

$$
\operatorname{Mod} \Gamma=\{\mathscr{A}:(\forall \lambda \in \Gamma) \mathscr{A} \vDash \lambda\} .
$$

Proposition 6.1. If $\Gamma$ is any class of limit laws, then $\operatorname{Mod} \Gamma$ is a wide variety.

Similarly for $\Sigma$ a set of ordinary laws, and $\Gamma$ as above, one can form $\operatorname{Mod}(\Sigma \cup \Gamma)=\operatorname{Mod} \Sigma \cap \operatorname{Mod} \Gamma$, which is again a wide variety. The main result of this section is the converse proposition.

THEOREM 6.2. Every wide variety is $\operatorname{Mod}(\Sigma \cup \Gamma)$ for some set $\Sigma$ of ordinary laws and some class $\Gamma$ of limit laws. [Burgin has claimed (1973) that $\Gamma$ is unnecessary here (even in a much more general setting than ours), but it should be evident that $\Gamma$ is needed, for a wide variety of reasons (e.g. the examples in $\$ 8$ and the results of $\$ 9)$.]

Proof. Take $X=$ the class of variables, and for convenience write $X=\cup\left\{X_{m}: m\right.$ any ordinal $\}$ with $X_{m}<X_{n}(m<n)$ and $\left|X_{m}\right| \leqq|m|$. For each $\mathrm{m}$, let $F_{2} \cdot\left(X_{\mathrm{m}}\right)$ be the $\mathscr{V}$-free topological algebra on the discrete space $X_{\mathrm{m}}$ as constructed in 1.1 and 1.2 above, and let $\Gamma_{m}$ be the class of all limit laws $\tau_{d} \rightarrow \tau$ where each $\tau_{d}$ and $\tau$ are terms in the variables in $X_{m}$ and $F_{i}\left(X_{m}\right)$ satisfies the limit law $\tau_{d} \rightarrow \tau$ under the identical assignment $\theta: X_{m} \rightarrow X_{m}$ (i.e. $\theta(x)=x$ ), in other words all limit laws $\tau_{d} \rightarrow \tau$ which are facts about convergence in $F_{r}\left(X_{m}\right)$. We now set

$$
\Gamma=\bigcup_{m \in \text { Ord }} \Gamma_{m}
$$

and let $\Sigma=\mathrm{Eq}$ Th $\mathscr{V}$, the set of ordinary equations true in $\mathscr{V}$. We claim that

$$
\mathscr{V}=\operatorname{Mod}(\Sigma \cup \Gamma)
$$

To check $\subseteq$, we really need only check that if $\mathscr{A} \in \mathscr{V}$, then $\mathscr{A} \vDash \tau_{d} \rightarrow \tau$ for $\left(\tau_{d} \rightarrow \tau\right) \in \Gamma_{m}$. And so we consider some assignment $\theta: X_{m} \rightarrow \mathscr{A}$. Clearly $\theta$ extends to a continuous homomorphism $\bar{\theta}: F_{1}\left(X_{m}\right) \rightarrow \mathscr{A}$. The continuity of $\bar{\theta}$ immediately tells us that $\mathscr{A}$ satisfies $\tau_{d} \rightarrow \tau$ under $\theta$.

To see the reverse inclusion and thus firish the proof, we will assume that

$$
\mathscr{A} \vDash \Sigma \in \Gamma
$$

and prove that $\mathscr{A} \in \mathscr{V}$. Choosing $\mathrm{m} \geqq|\mathscr{A}|$, we have surjective $\theta: X_{\mathrm{m}} \rightarrow \mathscr{A}$. But clearly, since $\mathscr{A} \models \Sigma$, by 0.3 we have $\overline{\mathscr{A}} \in \bar{\gamma}$ (indiscrete algebra and variety), and so $\theta$ can be extended to a homomorphism $\bar{\theta}: F_{\bar{r}}\left(\bar{X}_{\mathrm{m}}\right) \rightarrow \overline{\mathscr{A}}$. But since $F_{\bar{r}}\left(\bar{X}_{m}\right)=\overline{F_{1} \cdot\left(X_{m}\right)}$ (by 1.3$)$, we may regard $\bar{\theta}$ as a homomorphism 
$F_{\imath}\left(X_{\mathrm{m}}\right) \rightarrow \mathscr{A}$. Now the validity of $\Gamma$ in $\mathscr{A}$ (under the assignment $\theta$ ) asserts the continuity of $\bar{\theta}$. Thus $\mathscr{A} \in \boldsymbol{H}\left\{F_{1},\left(X_{m}\right)\right\}$, and so $\mathscr{A} \in \mathscr{V}$.

The above result may be compared with the results of Edgar (1973), who gave a way of defining the class of all topological spaces by some infinitary analogs of equations. His basic framework was somewhat different from ours, however. It is perhaps possible to combine the two theories, yielding every wide variety as definable by equations (with no mention of topological spaces or algebras, per se), but we do not now see any practical or advantageous way of doing so.

For some other extensions of Birkhoff's Theorem outside the realm of pure algebra, the reader is referred to Dixon (1976) and Bloom (1976). For Banach Algebras, Dixon considers "equations" of the form

$$
\left\|\tau\left(x_{1}, \cdots, x_{n}\right)\right\| \leqq k
$$

where $\tau$ is a term, $k$ is a fixed non-negative real and $\|\cdot\|$ denotes norm. (An ordinary equation $\sigma=\tau$ is of course expressible as

$$
\|\sigma-\tau\| \leqq 0 .)
$$

For ordered algebras, Bloom considers "inequalities" of the form

$$
\sigma\left(x_{1}, \cdots, x_{n}\right) \leqq \tau\left(x_{1}, \cdots, x_{n}\right) .
$$

(An ordinary equation $\sigma=\tau$ is of course expressible as the conjunction of the two inequalities

$$
\sigma \leqq \tau \quad \text { and } \quad \tau \leqq \sigma .)
$$

In dealing with laws, one also expects to have rules of deduction, analogous to Birkhoff's rules of proof for ordinary equations. We have not pursued this question in detail. We note that in addition to the obvious analogs of Birkhoff's rules, there would be a rule corresponding to the law of iterated limits; see p. 69 of Kelley (1955).

\section{Contingent limit laws}

Now we give a way of describing all varieties; it is not as nice as the description in $\$ 6$ of wide varieties, for there is no known way to see which classes of contingent limit laws actually define wide varieties. It is hoped that this situation will soon be rectified, either by a characterization of those classes of contingent limit laws which are $\boldsymbol{Q}$-closed, or by a modification of the notion so as to make all such classes $\boldsymbol{Q}$-closed. Possibly the recent category-theoretic study of theorems of this type by Banaschewski and Herrlich (1976) will be helpful here. 
Take $X$ and $f$ as in $\S 6, I$ any set, for each $i \in I$ a directed set $\left(D_{i}, \leqq\right)$, and assume that all $x_{d}^{i}\left(i \in I, d \in D_{i}\right)$ and $x^{i}(i \in I)$ are distinct members of $X$. A contingent limit law is an infinitary formal expression $\lambda$ of the form

$$
\left(\wedge_{i \in I} x_{d}^{i} \rightarrow x^{i}\right) \Rightarrow\left(\tau_{d} \rightarrow \tau\right)
$$

A topological algebra $\mathscr{A}$ satisfies the contingent limit law $\lambda$ under the assignment $\theta: X \rightarrow \mathscr{A}$ if:

$$
\tau_{d}[\theta] \rightarrow \tau[\theta]
$$

in the topology of $\mathscr{A}$ if for each $i \in I$

$$
\theta\left(x_{d}^{i}\right) \rightarrow \theta\left(x^{i}\right)
$$

in the topology of $\mathscr{A}$. And as usual,

$$
\mathscr{A} \vDash \lambda
$$

means that $\mathscr{A}$ satisfies $\lambda$ under every assignment, and for $\Gamma$ a class of contingent limit laws,

$$
\operatorname{Mod} \Gamma=\{\mathscr{A}:(\forall \lambda \in \Gamma) \mathscr{A} \vDash \lambda\} .
$$

THEOREM 7.1. If $\mathscr{V}$ is any variety, then there exists a class $\Gamma$ of contingent limit laws and a set $\Sigma$ of ordinary laws such that $\mathscr{V}=\operatorname{Mod}(\Sigma \cup \Gamma)$.

Proof. Let $X$ be the class of variables (as in $\S 6$ ). Clearly we may assume that $X=\cup X_{m}$ (m any cardinal) and for each $m$ there exists $Y$ with $|Y|=m$, such that $X_{\mathrm{m}}=Y \times Y \times \beta Y$ with $\beta Y$ the set of ultrafilters on $Y$. We now topologize $X_{\mathrm{m}}$ by regarding $X_{\mathrm{m}}$ as $Y \times \beta Y$ disjoint copies of $Y$ and topologizing each copy separately. In the $(y, \mu)$ th copy, we let the ultrafilter $\mu$ converge to $y$ and let no other ultrafilters converge (except for principal filters $\{U: x \in U\}$, which must converge to $x$ ). (Such a space certainly exists; in the case of $\mu$ non-principal it is a subspace of $\beta Y$ with the usual topology; in the case of $\mu$ principal, it is either discrete or an easily defined non- $T_{0}$ space.) We now take the free algebra $F_{v}\left(X_{m}\right)$ and let $\Gamma_{m}$ be the set of all contingent limit laws $\lambda$ of the form

$$
\left(\bigwedge_{i \in I} x_{d}^{i} \rightarrow x^{i}\right) \Rightarrow\left(\tau_{d} \rightarrow \tau\right)
$$

where $I$ is a set of convergent nets describing the topology of $X_{m}$, and $\tau_{d} \rightarrow \tau$ is a convergent net in the topology of $F_{V}\left(X_{m}\right)$. We now set

$$
\Gamma=\cup \Gamma_{m}
$$


and let $\Sigma=$ EqTh $\mathscr{V}$, the set of ordinary equations true in $\mathscr{V}$. We claim that

$$
\mathscr{V}=\operatorname{Mod}(\Sigma \cup \Gamma)
$$

To check $\subseteq$, we need only see that if $\mathscr{A} \in \mathscr{V}$, then $\mathscr{A} \vDash \lambda$ for every $\lambda \in \Gamma$. For $\lambda$ as above, we consider some assignment $\theta: X_{m} \rightarrow \mathscr{A}$. Now if

$$
\theta\left(x_{d}^{i}\right) \Rightarrow \theta\left(x^{i}\right)
$$

for each $i \in I$, in the topology of $\mathscr{A}$, then $\theta$ is continuous. And so there exists a continuous homomorphism $\bar{\theta}: F_{V}\left(X_{\mathrm{m}}\right) \rightarrow \mathscr{A}$ extending $\theta$. And so clearly $\bar{\theta}\left(\tau_{d}\right)=\tau_{d}[\theta]$ approaches $\bar{\theta}(\tau)=\tau[\theta]$ in $\mathscr{A}$, and so $\mathscr{A} \vDash \lambda$.

To see the reverse inclusion and thus finish the proof, we will assume that

$$
\mathscr{A} \vDash \Sigma \cup \Gamma
$$

and prove that $\mathscr{A} \in \mathscr{V}$. We first note that, if we take $m \geqq|\mathscr{A}|$, there exists a quotient map of spaces, $\theta: X_{\mathrm{m}} \rightarrow \mathscr{A}$. (One simply makes each convergent ultrafilter on $\mathscr{A}$ the image of an isomorphic convergent ultrafilter on $X_{\mathrm{m}}$; the unneeded parts of $X_{m}$ are mapped to a point.) As before, if $\bar{V}$ is the indiscrete part of $\mathscr{V}$, there exists a homomorphism $F_{\tilde{V}}\left(\bar{X}_{m}\right) \rightarrow \bar{A}$ extending $\theta$, i.e. $\bar{\theta}: \bar{F}_{\mathrm{v}}\left(\bar{X}_{\mathrm{m}}\right) \rightarrow \bar{A}$. But the continuity of $\bar{\theta}$ is simply expressed by $\Gamma_{m}$ (with this assignment $\theta)$. Since we took $\theta$ to be a quotient map, we see that $\mathscr{A} \in \boldsymbol{Q}\left\{F_{V}\left(X_{m}\right)\right\}$, and hence, since $\mathscr{V}$ is a variety, $\mathscr{A} \in \mathscr{V}$.

Unfortunately, not every class defined by contingent limit laws is a variety. (An example was kindly supplied to the author by P. G. Dixon [private communication]. His example has four unary operations and no other operations.) A class defined by contingent limit laws is easily seen to be $\boldsymbol{S P}$-closed; the difficulty arises with $\boldsymbol{Q}$. There are occasional ways around this, as we will see. Thus, so far, our main use of Theorem 7.1 is in suggesting a form for external description of varieties. See the next section for examples.

Proposition 7.2. If $\lambda$ is a contingent limit law of the form

$$
\left(x_{n} \rightarrow x\right) \Rightarrow \tau_{d} \rightarrow \tau
$$

(with $n$ ranging over natural numbers), and if $\mathscr{A} \vDash \lambda$, where $\mathscr{A}$ is first-countable, then $\lambda$ holds in every open homomorphic image of $\mathscr{A}$.

Proof. Let $f: \mathscr{A} \rightarrow \mathscr{B}$ be an open continuous homomorphism, and suppose $b_{n} \rightarrow b$ in the topology of $\mathscr{B}$. Select $a \in \mathscr{A}$ with $f(a)=b$ and let

$$
\mathscr{A}=U_{1} \supseteq U_{2} \supseteq U_{3} \supseteq \cdots
$$

be an open neighborhood basis at $a$. Thus each $f\left[U_{i}\right]$ is open, and so there exists $N(i)$ so that $b_{n} \in f\left[U_{i}\right]$ for $n \geqq N(i)$. We may clearly assume $N(i+1) \geqq$ 
$N(i)$, and $N(1)=1$. We now choose a sequence $a_{1}, a_{2}, \cdots$ by saying that $f\left(a_{n}\right)=b_{n}$ and $a_{n} \in U_{i}$ where $i$ is the greatest $i$ for which $n \geqq N(i)$. It is clear that $a_{n} \rightarrow a$. If $y$ is any variable not among $x_{1}, x_{2}, \cdots$, assign it a value in $\mathscr{A}$ which is an $f$-preimage of its assigned value in $\mathscr{B}$. Now it is immediate that $\tau_{d}\left[a_{1}, a_{2}, \cdots\right] \rightarrow \tau\left[a_{1}, a_{2}, \cdots\right]$ in $\mathscr{A}$, and so, by continuity of $f$, $\tau_{d}\left[b_{1}, b_{2}, \cdots\right] \rightarrow \tau\left[b_{1}, b_{2}, \cdots\right]$ in $\mathscr{B}$.

The following useful corollary was pointed out to me by S. A. Morris.

COROLlary 7.3. If $\lambda$ is a contingent limit law of the form

$$
\left(x_{n} \rightarrow x\right) \Rightarrow \tau_{d} \rightarrow \tau
$$

(with $n$ ranging over natural numbers) and $\Omega$ is a collection of first-countable algebras from some congruence-permutable variety such that

$$
\mathscr{A} \vDash \lambda
$$

for every $\mathscr{A} \in \Omega$, then $\lambda$ holds in $Q S P \Omega$.

Proof. We apply Corollary 4.2. Since $\lambda$ obviously holds in any indiscrete algebra, it is enough to check that $\lambda$ holds in $\bar{Q} \bar{S} \boldsymbol{F} \Omega$. But this follows from Proposition 7.2 .

\section{Examples of (contingent) limit laws}

For spaces (i.e. topological algebras $\left(A, \mathcal{U}, F_{1}\right)_{t \in T}$ with $T=\varnothing$ ), we have the limit law

$$
x \rightarrow y,
$$

defining the variety of indiscrete spaces (here $x$ denotes a constant net). In fact there are no other interesting contingent limit laws of this type, for by a result of Kannan (1972; Theorem F) there are only three varieties of spaces: all spaces, all indiscrete spaces and all singleton spaces. [Here our theory differs considerably from that of Edgar (1973), who found "equational" definitions for various classes of spaces, e.g. the class of Hausdorff spaces.]

Semilattices are given by the laws

$$
\begin{gathered}
x(y z)=(x y) z \\
x y=y x \\
x x=x .
\end{gathered}
$$

Aside from the above law for indiscreteness, we have the limit laws 


$$
\begin{aligned}
& x \rightarrow x y \\
& x y \rightarrow y
\end{aligned}
$$

(where the left hand sides are constant nets). If we take $x \leqq y$ in a semilattice to mean that $x y=x$, then the first law asserts that if $U$ is open and $x \geqq y \in U$, then $x \in U$. The second states that if $U$ is open and $x \leqq y \in U$, then $x \in U$. We will see that every non-trivial limit law for semilattices is equivalent to one of these two. Thus we consider

$$
\tau_{d} \rightarrow \tau
$$

where each $\tau_{d}$ is a semilattice product of variables, and, w.l.o.g. $\tau=x_{1} \cdots x_{n}$. Now if frequently $\tau_{d}$ has no factor among $x_{1}, \cdots, x_{n}$, then by an obvious substitution into a subnet, we deduce

$$
x \rightarrow y,
$$

from which all limit laws follow. Thus we may assume that eventually every $\tau_{d}$ has some $x_{i}$ as a factor $(1 \leqq i \leqq n)$. We next note that either

(a) frequently $\tau_{d}$ has a factor other than $x_{1}, \cdots, x_{n}$; or

(b) eventually all factors of $\tau_{d}$ are among $x_{1}, \cdots, x_{n}$.

By some simple substitutions in (a), we see that either

(a') $\tau_{d} \rightarrow \tau$ implies $x y \rightarrow y$; or

(b) eventually all factors of $\tau_{d}$ are among $x_{1}, \cdots, x_{n}$.

By successive examination of the sets $\left\{d: x_{i}\right.$ is not a factor of $\left.\tau_{d}\right\}$, we see that either

(c) for some $i(1 \leqq i \leqq n)$, frequently $x_{i}$ is not a factor of $\tau_{d}$; or

(d) eventually $x_{1} \cdots x_{n}$ is a factor of $\tau_{d}$.

Again by substitution we see that either

(c') $\tau_{d} \rightarrow \tau$ implies $x \rightarrow x y$; or

(d) eventually $x_{1} \cdots x_{n}$ is a factor of $\tau_{d}$.

We may now simply examine cases:

(a') and ( $\left.\mathrm{c}^{\prime}\right): \tau_{d} \rightarrow \tau$ is equivalent to $x \rightarrow y$

(a') and (d): $\tau_{d} \rightarrow \tau$ is equivalent to $x y \rightarrow y$

(b) and ( $\left.\mathrm{c}^{\prime}\right): \tau_{d} \rightarrow \tau$ is equivalent to $x \rightarrow x y$

(b) and (d): $\tau_{d} \rightarrow \tau$ is equivalent to $x \rightarrow x$.

It is usually difficult or impossible to find an explicit set of limit laws defining a given wide variety. We will do this for the rare case we can, namely $\mathscr{V}=\boldsymbol{H S P 2}$, where $\mathbf{2}$ denotes a discrete group of two elements written additively. We will exhibit a set $\Gamma$ of limit laws which (together with the laws for groups of exponent two) will define this variety $\mathscr{V}$. Fixing temporarily a cardinal $m$, consider the infinite matrix 


$$
M_{\mathrm{m}}=\left[\begin{array}{ccccc}
\varphi_{0}(0) & \varphi_{0}(1) & \varphi_{0}(2) & \cdots & \\
\varphi_{1}(0) & \varphi_{1}(1) & \varphi_{1}(2) & \\
\varphi_{2}(0) & \varphi_{2}(1) & \varphi_{2}(2) & \\
\cdot & & \cdot & \\
\cdot & & & \cdot & \\
& & & \varphi_{\eta}(\alpha) \\
& & & & \cdot
\end{array}\right]
$$

where $\alpha$ ranges over ordinals $<\mathrm{m}$ and $\varphi_{0}, \varphi_{1}, \cdots, \varphi_{\eta}, \cdots$ enumerate all functions $m \rightarrow 2$. Define $D_{m}$ to be the set of all finite submatrices of $M_{m}$ whose rows all sum to zero $(\bmod 2)$. Each member of $D_{m}$ looks like

$$
\left(\varphi_{\eta}(\alpha)\right)_{\alpha \in J, \eta \in F}
$$

for some finite $J$ and $F$; we may denote it briefly by $[J, F]$. Elementary linear algebra establishes that for every finite $F$ there exists non-empty finite $J$ with $[J, F] \in D_{m}$. (In other words, for every finite set of rows of $M_{m}$ we can find a finite set of columns so that in the resulting finite submatrix, all rows add to zero $(\bmod 2)$ ) $)$ Order $D_{\mathrm{m}}$ by inclusion on the second coordinate, namely $[J, F]<\left[J^{\prime}, F^{\prime}\right] \leftrightarrow F \subset F^{\prime}$. We now take variables $x_{\alpha}(\alpha<m)$ and define

$$
\tau_{[J, F]}=\sum_{\alpha \in J} x_{\alpha}
$$

It is not difficult to see that 2 obeys the limit law

$$
\tau_{[J, F]} \rightarrow 0,
$$

for if $\varphi:\left\{x_{\alpha}\right\} \rightarrow 2$ is any assignment, then for some $\eta, \varphi\left(x_{\alpha}\right)=\varphi_{\eta}(\alpha)$ and under this assignment $\tau_{[J, F]}$ receives the value 0 whenever $\mathrm{F} \supseteq\left\{\varphi_{\eta}\right\}$.

To finish this example, we will establish that every group of exponent two obeying (*) (for every $m$ ) is in HSP2. Thus we may assume that $\mathscr{A}$ is a group of exponent two in which (*) holds. It is, in fact, enough to assume that $(*)$ holds for $\mathrm{m}=|\mathscr{A}|$. There exists a natural homomorphism

$$
\theta: F(\mathscr{A}) \rightarrow \mathscr{A}
$$

where $F(\mathscr{A})$ denotes the free group of exponent two on $\mathscr{A}$, embedded in the standard manner as the subgroup of $2^{\left(\mathbf{2}^{\star A}\right)}$ generated by all $\hat{a}(a \in \mathscr{A})$, where $\hat{a}(\varphi)=\varphi(a)$. Thus the discrete topology on 2 induces a topology on $F(\mathscr{A})$, and so it will be enough to show that $\theta$ is continuous at 0 . Let $E$ be the usual directed set

$$
E=\{(x, U): x \in U \in N\}
$$


where $N$ is the neighborhood basis of 0 consisting of all sets of the form $F(\mathscr{A}) \cap\left\{\lambda \in \mathbf{2}^{\left.(2)^{\star x}\right)}: \lambda\left(\varphi_{1}\right)=\cdots=\lambda\left(\varphi_{k}\right)=0\right\}$, with $E$ ordered by inclusion on the second coordinate. Define

$$
a: E \rightarrow F(\mathscr{A})
$$

by first coordinate projection. To check continuity of $\theta$ at 0 , it is enough to check that the net $\theta\left(a_{e}\right)$ converges to 0 in $\mathscr{A}$. But $E$ is obviously isomorphic to $D_{\mathrm{m}}$ for $\mathfrak{m}=|\mathscr{A}|$, and with this $\mathrm{m}$ we have $\theta\left(a_{e}\right)=\tau_{e}[1]$, where 1 is the identity assignment $\mathscr{A} \rightarrow \mathscr{A}$. And so $(*)$ asserts that $\theta\left(a_{\mathrm{e}}\right)$ converges to 0 .

Turning now to limit laws on some familiar groups, we let $\mathbb{R}$ denote the reals with the usual topology $\mathbb{T}=\mathbb{R} / \mathbb{Z}$ the circle group and $\mathbb{Q} / \mathbb{Z}$ its torsion subgroup. We note that $\mathbb{Q} / \mathbb{Z}$ obeys the (additively written) limit law [a similar law was known earlier to Edgar]

$$
m ! x \rightarrow 0,
$$

but $\pi$ does not - as seen by taking e.g.

$$
x=\sum_{k=0}^{\infty} \frac{\left[\frac{1}{3} k\right]}{k !}
$$

(and thus $m ! x \rightarrow \frac{1}{3}$ ). And so limit laws are not preserved under forming closures. (Cf. also Brooks, Morris, Saxon (1973), remarks on top of p. 194.) And we observe that this is an easy proof that

$$
\mathbb{T} \notin \boldsymbol{H S P}\{\boldsymbol{Q} / \mathbb{Z}\} .
$$

But we shall now see that there exists a net [Loxton showed that $n_{d}$ cannot be a sequence] of positive integers $n_{d}$ such that

$$
\mathbb{T} \vDash n_{d} x \rightarrow 0 \text {. }
$$

To see this, let $D$ be the set of all $(N, n, \alpha, p)$, where $N$ and $n$ are positive integers and for some $k, \alpha=\left(\alpha_{1}, \cdots, \alpha_{k}\right) \in \mathbb{R}^{k}$ and $p=\left(p_{1}, \cdots, p_{k}\right) \in \mathbb{Z}^{k}$, such that

$$
\left|n \alpha_{i}-p_{i}\right| \leqq N^{-1} \quad(i=1, \cdots, k) .
$$

Order $D$ by saying $(N, n, \alpha, p)<\left(N^{\prime}, n^{\prime}, \alpha^{\prime}, p^{\prime}\right)$ iff $\left\{\alpha_{1}, \cdots, \alpha_{k}\right\} \subseteq\left\{\alpha_{1}^{\prime}, \cdots, \alpha_{k}^{\prime}\right\}$ and $N^{\prime}>N$. Dirichlet's 1842 theorem of simultaneous rational approximation of reals (see e.g. Schmidt (1971, p. 216)) asserts that

$$
\forall N \alpha \exists n p[(N, n, \alpha, p) \in D],
$$

which clearly implies that $D$ is directed. Now for $d=(N, n, \alpha, p) \in D$, we define $n_{d}=n$. It should be clear that 


$$
n_{d} x \rightarrow 0
$$

in $\pi$. As a corollary, we observe that

\section{$\mathbb{Z} \notin \boldsymbol{H S P}(\mathbb{T})$,}

a fact earlier observed by Morris (1970b, Theorem 5.4). Morris also observed that

$$
\mathscr{G} \vDash n_{d} x \rightarrow 0
$$

for any compact group $\mathscr{G}$ (since it holds in any orthogonal group by one-parameter subgroups). But the limit law $n_{d} x \rightarrow 0$ also holds in some groups which are not in the wide variety generated by all compact groups, e.g. any discrete torsion group of power $>2^{\aleph_{0}}$ (by 3.4, 4.1 and 4.5).

Morris (1970b, Example 3.2) mentions Looker's example of the variety $\mathscr{V}$ of all topological groups $\mathscr{G}$ such that the commutator subgroup of $\mathscr{G}$ is contained in every neighborhood of the identity. Clearly $\mathscr{V}$ is defined by the limit law

$$
x^{-1} y^{-1} x y \rightarrow 1
$$

(where the L.H.S. denotes a constant net). Similar remarks apply to any other verbal subgroup.

As Morris has pointed out, if one considers the wide variety $\mathscr{V}$ of groups defined by the limit law

$$
\left[\left[\cdots\left[\left[x_{1}, x_{2}\right], x_{3}\right], \cdots\right], x_{n}\right] \rightarrow 1
$$

(with $[$,$] denoting commutator), then the discrete groups in \mathscr{V}$ are precisely the nilpotent groups. Clearly similar limit laws can be found for any directed union of finitely based varieties of groups (or subvarieties of any congruenceregular variety, using Csákány's polynomials of $\$ 3$ ). Even more is true. If $\boldsymbol{K}$ is any directed family of (algebraic) subvarieties of some variety with regular and permutable congruences, then by $\$ \$ 3$ and 4 , if we equip each algebra in $\cup \boldsymbol{K}$ with the discrete topology, then the discrete algebras in $\boldsymbol{Q S P}(\cup \boldsymbol{K})$ are precisely those in $\cup \boldsymbol{K}$.

Turning to contingent limit equations, we again look at group theory, and examine the anti-Archimedean law, written additively as [Strictly speaking this is not a contingent limit law, since 0 is not a variable, but clearly it could be modified to be a contingent limit law]

$$
\left(x_{n} \rightarrow 0\right) \Rightarrow\left(n x_{n} \rightarrow 0\right) .
$$

It is obvious that $\mathbb{Z}$ obeys (AA) but $\mathbb{T}$ does not [A nother interesting class of groups satisfying (AA) is that of all proper enlargements of the reals (see e.g. Robinson (1966))], and thus by Corollary 7.3, 


$$
\text { QSP } \mathbb{Z} \vDash(\mathrm{AA}),
$$

and so we see that

$$
\pi \notin \boldsymbol{Q S P}\{\mathbb{Z}\},
$$

a fact earlier observed by Morris (1974, Theorem 3). Morris has also observed that the contingent limit laws

$$
\left(x_{d} \rightarrow 1\right) \Rightarrow\left(z_{d} x_{d} z_{d}^{-1} \Rightarrow 1\right)
$$

(with $d$ ranging over an arbitrary directed set $D$ ) define the variety of S.I.N. groups (groups with small invariant neighborhoods, see Morris (1973)). We omit the proof.

Returning to general algebra for a moment before looking at topological vector spaces, we consider topological algebras $\left(A, \mathcal{U}, F_{t}\right)_{t \in T}$ of type $\left(n_{t}\right)_{t \in T}$, and for simplicity we wish to assume that all $n_{t}$ are equal, say $n_{t}=n(\forall t \in T)$. We wish to examine the case in which $T$ itself is a topological space, and we consider the class $\mathscr{V}$ of those algebras for which

$$
F: T \times A^{n} \rightarrow A
$$

is continuous (where $F(t, \alpha)=F_{t}(\alpha)$ ). (This class has been studied by Burgin (1973) and Boardman and Vogt (1973).) In fact it should be clear that $\mathscr{V}$ is defined by the contingent limit laws

$$
\left(\bigwedge_{i=1}^{n} x_{d}^{i} \rightarrow x^{i}\right) \Rightarrow\left(F_{t_{d}}\left(x_{d}^{1}, \cdots, x_{d}^{n}\right) \rightarrow F_{1}\left(x^{1}, \cdots, x^{n}\right)\right),
$$

where $t_{d} \rightarrow t$ ranges over all convergent nets in $T$. One easily checks directly from the definitions or from these contingent limit laws that $\mathscr{V}$ is a variety if $\mathscr{V}$ has permutable congruences.

As a special case of the preceding, we have the variety $\mathscr{V}$ of topological vector spaces (over the real topological field $\mathbb{R}$ ), which are vector spaces

$$
(A,+, \bar{\lambda})_{\lambda \in \boldsymbol{H}}
$$

such that $(\lambda, x) \mapsto \bar{\lambda}(x)=\lambda x$ is continuous on $\mathbb{R} \times A$. We will now give a class $\Gamma$ of contingent limit laws which defines the subvariety of locally convex spaces. For $D$ any directed set, we take $\bar{D}=\omega \times D$, ordered by the second coordinate, and we take variables $x_{n d}((n, d) \in \bar{D})$. By a convex combination, we understand a term $\tau=\Sigma_{i-1}^{k} \lambda_{i} x_{i}$ with $\Sigma \lambda_{i}=1$ and each $\lambda_{i} \geqq 0$. For $D$ a directed set, we take $\overline{\bar{D}}=L \times D$, where $L$ is the set of linear combinations, ordered by the second coordinate, and for $e=(\tau, d)=\left[\sum_{i=1}^{k} \lambda_{i} x_{i}, d\right] \in \overline{\bar{D}}$, we put $\tau_{e}=\sum_{i=1}^{k} \lambda_{i} x_{i d}$. We now take $\Gamma$ to be the class of all contingent limit laws

$$
\left(x_{n d} \rightarrow 0\right) \Rightarrow\left(\tau_{e} \rightarrow 0\right),
$$


with $D$ (hence $\bar{D}$ and $\overline{\bar{D}}$ ) ranging over all directed sets. We claim that $\mathscr{V} \cap \operatorname{Mod} \Gamma$ is precisely the class of locally convex topological vector spaces (where $\mathscr{V}$ is the class of all topological vector spaces). (A vector space is locally convex if every neighborhood of 0 contains a convex neighborhood of 0 . For the fact that this class is a variety, see e.g. Diestel, Morris and Saxon (1972).) Let us first check that if $\mathscr{A}$ is locally convex, then $\mathscr{A} \vDash \Gamma$. Then (omitting mention of the assignment) we need to prove that $\tau_{e} \rightarrow 0$ (given $x_{n d} \rightarrow 0$ ), i.e. that given a neighborhood $U$ of $0, \tau_{e}$ is eventually in $U$. But $U$ contains a convex neighborhood $V$, and $x_{n d}$ is eventually in $V$, i.e. $x_{n d} \in V$ for $d>d_{0}$. But then clearly $\tau_{e} \in V \subseteq U$ for $e=(\tau, d)$ with $d>d_{0}$.

Conversely, we must show that every model of $\Gamma$ is locally convex. For $\mathscr{A} \vDash \Gamma$, we let $N$ be the full neighborhood system at 0 and take

$$
D=\left\{\left(U, a_{0}, a_{1}, \cdots\right): U \in N,(\forall i) a_{i} \in U\right\},
$$

ordered by reverse inclusion on the first coordinate, and define the assignment $\theta:\left\{x_{n d}\right\} \rightarrow \mathscr{A}$ via $\theta\left(X_{n d}\right)=a_{n}$ for $d=\left(U, a_{0}, a_{1}, \cdots\right)$. A standard argument tells us that $\theta\left(x_{n d}\right) \rightarrow 0$, and hence, by $\Gamma, \tau_{e}[\theta] \rightarrow 0$. Now to check local convexity, we look at an arbitrary neighborhood $U$ of 0 . Since $\tau_{e}[\theta]$ is eventually in $U$, there exists an open neighborhood $V$ so that $\tau_{e}[\theta] \in U$ for any $e=\left(\sum \lambda_{i} x_{i},\left(W, a_{0}, a_{1}, \cdots\right)\right)$ with $W \subseteq V$, and $a_{0}, a_{1}, \cdots$ arbitrary $\in W$. Thus in fact $\tau_{e}[\theta]=\Sigma \lambda_{i} a_{i} \in U$ for $a_{0}, a_{1}, \cdots$ arbitrary in $V$, i.e. $U \supseteq$ the convex hull of $V$, which is a neighborhood of 0 .

Another natural example of a variety of topological algebras with a continuous system of operations is provided by the class $\mathscr{V}$ of topological algebras which obey a law $\lambda$ within homotopy. E.g. if $\lambda$ is the commutative law, then we may take an operation $F$ and a continuous family $F_{t}$ of operations $(0 \leqq t \leqq 1)$ such that

$$
\begin{aligned}
& F_{0}(x, y)=F(x, y) \\
& F_{1}(x, y)=F(y, x) .
\end{aligned}
$$

Such families of operations are discussed in Boardman and Vogt (1973).

\section{The number of topological varieties}

We give two different theorems extending Morris's result (1970b) that there are a proper class of varieties with underlying algebraic variety a fixed variety of groups.

THEOREM 91 . If $\mathscr{V}$ is a variety of algebras with regular and permutable 
congruences, then there exists a proper class of topological varieties with $\mathscr{V}$ as underlying variety of algebras.

Proof. For each infinite cardinal $m \geqq|T|$ (where the type is $\left.\left(n_{t}\right)_{t \in T}\right)$, let $\mathscr{V}_{m}$ be the variety generated by $F_{V}(m)$ with the discrete topology. By Theorem 4.1 any discrete $\mathscr{A} \in \mathscr{V}_{\mathrm{m}}$ must be in $\boldsymbol{S P} \overline{\boldsymbol{Q}} \overline{\boldsymbol{S}} \boldsymbol{F}\left\{\boldsymbol{F}_{\boldsymbol{V}}(\mathrm{m})\right\}$, and so by Theorem $3.2 \mathscr{A}$ must be in $\boldsymbol{S F} \bar{Q} \bar{S} \boldsymbol{F}\left\{F_{V}(\mathrm{~m})\right\}$, and so $|\mathscr{A}| \leqq \mathrm{m}$. And so

$$
\mathscr{V}_{\mathrm{m}} \neq \mathscr{V}_{\mathrm{n}}
$$

whenever $m \neq n$.

Recall that a variety is residually small (see Taylor (1972)) if its subdirectly irreducible algebras form a set.

THEOREM 9.2. If $\mathcal{V}$ is a variety of algebras which is congruence-3permutable and not residually small, then there exists a proper class of topological wide varieties with $\mathscr{V}$ as underlying variety of algebras.

Proof. For each infinite cardinal $m \geqq|T|$, let $\mathscr{A}_{m}$ be a subdirectly irreducible algebra in $\mathscr{V}$ of power $m$, equipped with the discrete topology, and let

$$
\mathscr{V}_{\mathrm{m}}=\boldsymbol{H S P}\left\{\mathscr{A}_{\mathrm{m}}, \mathscr{A}\right\}
$$

where $\mathscr{A}=F_{\nu}\left(\boldsymbol{N}_{0}\right)$ with the indiscrete topology. It is immediate from Theorem 4.3 that if $\mathfrak{m}<\mathrm{n}$, then $\mathscr{A}_{\mathfrak{n}} \notin \mathscr{V}_{\mathfrak{m}}$. Thus the varieties $\mathscr{V}_{\mathrm{m}}$ are all distinct.

Added in proof (7 April, 1977). I thank Ralph McKenzie for pointing out that the inequality in $1.5(3)$ is valid only if the summation is taken over distinct pairs $\left(x_{i}, y_{i}\right)$. This does not affect the application of 1.5 to the proof of Theorem 2.1.

This article has a sequel, 'Primal topological algebras,' to appear in Algebra Universalis, in which we consider the variety generated by a single algebra which has all operations compatible with a given space.

Our results of $\$ \$ 6,7$ are re-proved and elucidated in P. G. Dixon, 'Classes of algebraic systems defined by universal Horn sentences,' to appear in Algebra Universalis. Here one will also find Dixon's example mentioned before 7.2 .

For wide varieties of topological semigroups, see page 17 of K. H. Hofmann (1976), 'Topological semigroups-History, theory, applications,' Jber. Deutsch. Math.- Verein. 78, 9-59.

The special spaces used in the proof of Theorem 7.1 have appeared 
before in O. Fröhlich (1964), 'Das Halbordnungssystem der topologischen Räumen auf einer Menge,' Math. Annalen 156, 79-95, and in W.-M. Chan, D. Higgs and B. Lenihan (1974), 'Compactness as a type of injectivity,' Gen. Topology and Applic. 4, 103-107.

\section{References}

J. T. Baldwin and J. Berman (to appear), 'A model-theoretical approach to Mal'cev conditions, J. Symbolic Logic.

B. Banaschewski and H. Herrlich (1976), 'Subcategories defined by implications,' Houston J. Math. 2, 149-171.

Garrett Birkhoff (1948 and 1967), Lattice Theory (Amer. Math. Soc. Colloquium Publications, 25. New York).

Stephen L. Bloom (1976), 'Varieties of ordered algebras,' J. Computer System Sci. 13, 200-212.

J. M. Boardman and R. M. Vogt (1973), Homotopy Invariant Algebraic Structures on Topological Spaces (Lecture Notes in Mathematics, 347. Springer-Verlag, Berlin, Heidelberg, New York, 1973).

M. S. Brooks, Sidney A. Morris and Stephen A. Saxon (1973), 'Generating varieties of topological groups,' Proc. Edinburgh Math. Soc. (2) 18, 191-197.

M. С. Бургин [M. S. Burgin] (1972), “Свободные топологнческне группы и универсальные алгебры" [Free topological groups and universal algebras], Dokl. Akad. Nauk SSSR 204, 9-11, Soviet Math. Dokl. 13, 561-564.

M. С. Бургин [M. S. Burgin] (1973), "Топологические алгебры с непрерывнымн системамн операций" [Topological algebras with continuous systems of operations], Dokl. Akad. Nauk SSSR 213, 505-508; Soviet Math. Dokl. 14, 1711-1715.

P. M. Cohn (1965), Universal Algebra (Harper's Series in Modern Mathematics. Harper and Row, New York, Evanston, London, 1965).

P. Crawley and R. P. Dilworth (1973), Algebraic Theory of Lattices (Prentice-Hall, Englewood Cliffs, 1973).

B. Csákány (1970), 'Characterizations of regular varieties,'Acta Sci. Math. (Szeged) 31, 187-189.

J. Diestel, Sidney A. Morris and Stephen A. Saxon (1972), 'Varieties of linear topological spaces, Trans. Amer. Math. Soc. 172, 207-230.

P. G. Dixon (1976), 'Varieties of Banach algebras,' Quart. J. Math. (Oxford) (2) 27, 481-487.

G. A. Edgar (1973), 'The class of topological spaces is equationally definable,' Algebra Universalis 3, 139-146.

G. D. Findlay (1960), 'Reflexive homomorphic relations,' Canad. Math. Bull. 3, 131-132.

J. A. Gerhard (1971), 'Subdirectly irreducible idempotent semigroups,' Pacific J. Math. 39, 669-676.

George Grätzer (1968), Universal Algebra (The University Series in Higher Mathematics. Van Nostrand, Princeton, New Jersey, Toronto, London, Melbourne, 1968).

G. Grätzer (1970), 'Two Mal'cev-type theorems in universal algebra,' J. Combinatorial Theory 8, 334-342.

J. Hagemann (to appear), 'On regular and weakly regular congruences,' Algebra Universalis.

J. Hagemann (preprint), 'Foundations of universal topological algebra I.'

Bjarni Jónsson (1953), 'On the representation of lattices,' Math. Scand. 1, 193-206.

V. Kannan (1972), 'Reflexive cum coreflexive subcategories in topology,' Math. Ann. 195, $168-174$.

J. L. Kelley (1955), General Topology (Van Nostrand, Princeton, New Jersey, 1955). 
А. И. Мальцев [А. I. Mal'cev] (1954), “К общей теории алгебраических систем" [On the general theory of algebraic systems], Mat. Sb. (NS) 35 (77), 3-20; Amer. Math. Soc. Transl. (2) 27 (1963), 125-142.

А. А. Марков [А. А. Markov] (1945), "О свободных топологических группах" [On free topological groups], Izv. Akad. Nauk SSSR Ser. Mat. 9, 3-64; Amer. Math. Soc. Transl. 30 (1950), 11-88; reprinted Amer. Math. Soc. Transl. (1) 8 (1962), 195-272.

Sidney A. Morris (1969), 'Varieties of topological groups,' Bull. Austral. Math. Soc. I, $145-160$.

Sidney A. Morris (1970a), 'Varieties of topological groups II,' Bull. Austral. Math. Soc. 2, $1-13$.

Sidney A. Morris (1970b), 'Varieties of topological groups III,'Bull. Austral. Math. Soc. 2, $165-178$.

Sidney A. Morris (1973), 'Varieties of topological groups generated by maximally almost periodic groups, Colloq. Math. 28, 47-50.

Sidney A. Morris (1974), 'Lie groups in varieties of topological groups,' Colloq. Math. 30, 229-235.

J. Mycielski (1964), ' $\alpha$-incompactness of $N^{\alpha}$ 'Bull. Acad. Polon. Sci. Sér. Sci. Math. Astronom. Phys. 12, 437-438.

Walter D. Neumann (1974), 'On Malcev conditions,' J. Austral. Math. Soc. 17, 376-384.

Abraham Robinson (1966), Non-standard Analysis (Studies in Logic and the Foundations of Mathematics, 11. North Holland. Amsterdam, 1966).

Wolfgang M. Schmidt (1971), 'Approximation to algebraic numbers,' Enseignement Math. (2) 17, 187-253.

S. Świerczkowski (1964), 'Topologies in free algebras,' Proc. London Math. Soc. (3) 14, 566-576.

Walter Taylor (1971), 'Some constructions of compact algebras,' Ann. Math. Logic 3, 395-435.

Walter Taylor (1972), 'Residually small varieties,' Algebra Universalis 2, 33-53.

Walter Taylor (1973), 'Characterizing Mal'cev conditions,' Algebra Universalis 3, 351-397.

Walter Taylor (1974), 'Uniformity of congruences,' Algebra Universalis 4, 342-360.

Walter Taylor (1975a), 'Topological laws for topological varieties,' Notices Amer. Math. Soc. 22, A618-A619, Abstract \# 75T-A227.

Walter Taylor (1975b), 'Varieties of topological algebras,' Notices Amer. Math. Soc. 22, A506, Abstract \# 75T-A185.

Walter Taylor (1976), 'Pure compactifications in quasi-primal varieties,' Canadian J. Math. 28, $50-62$.

Walter Taylor (to appear), 'Varieties obeying homotopy laws,' Canadian J. Math.

Rudolf Wille (1970), Kongruenzklassengeometrien (Lecture Notes in Mathematics, 113 Springer-Verlag, Berlin, Heidelberg, New York, 1970).

Department of Mathematics,

University of Colorado,

Boulder, Colorado 80309 ,

U.S.A. 